\title{
A UNIFIED APPROACH TO VARIOUS GENERALIZATIONS OF ARMENDARIZ RINGS
}

\author{
GREG MARKS ${ }^{\bowtie}$, RYSZARD MAZUREK and MICHAŁ ZIEMBOWSKI
}

(Received 5 February 2009)

\begin{abstract}
Let $R$ be a ring, $S$ a strictly ordered monoid, and $\omega: S \rightarrow \operatorname{End}(R)$ a monoid homomorphism. The skew generalized power series ring $R[[S, \omega]]$ is a common generalization of (skew) polynomial rings, (skew) power series rings, (skew) Laurent polynomial rings, (skew) group rings, and Mal'cev-Neumann Laurent series rings. We study the $(S, \omega)$-Armendariz condition on $R$, a generalization of the standard Armendariz condition from polynomials to skew generalized power series. We resolve the structure of $(S, \omega)$ Armendariz rings and obtain various necessary or sufficient conditions for a ring to be $(S, \omega)$-Armendariz, unifying and generalizing a number of known Armendariz-like conditions in the aforementioned special cases. As particular cases of our general results we obtain several new theorems on the Armendariz condition; for example, left uniserial rings are Armendariz. We also characterize when a skew generalized power series ring is reduced or semicommutative, and we obtain partial characterizations for it to be reversible or 2-primal.
\end{abstract}

2000 Mathematics subject classification: primary 16S99, 16W60; secondary 06F05, 16P60, 16S36, 16U80, 20M25.

Keywords and phrases: skew generalized power series ring, $(S, \omega)$-Armendariz, semicommutative, 2-primal, reversible, reduced, uniserial.

\section{Introduction}

In 1974 Armendariz noted in [3] that whenever the product of two polynomials over a reduced ring $R$ (that is, a ring without nonzero nilpotent elements) is zero, then the products of their coefficients are all zero, that is, in the polynomial ring $R[x]$ the following holds:

$$
\begin{aligned}
& \text { for any } f(x)=\sum a_{i} x^{i}, g(x)=\sum b_{j} x^{j} \in R[x], \\
& \text { if } f(x) g(x)=0, \text { then } a_{i} b_{j}=0 \text { for all } i, j .
\end{aligned}
$$

Nowadays the property $(*)$ is known as the Armendariz condition, and rings $R$ that satisfy $(*)$ are called Armendariz rings. The systematic study of Armendariz rings was

The second author was supported by Bialystok University of Technology grant W/WI/7/08, MNiSW grant N N201 268435, and KBN grant 1 P03A 03227.

(C) 2010 Australian Mathematical Publishing Association Inc. 0004-9727/2010 \$16.00 
initiated by Rege and Chhawchharia in 1997 in [37], and since then many results have been obtained on the structure of such rings, the behaviour of the Armendariz condition under ring constructions, and relationships of Armendariz rings to other significant classes of rings (see the references for some literature on the subject). As observed by Hirano in [12], the simple condition $(*)$ hides a remarkable connection between the right annihilators of $R$ and those of $R[x]$. Namely, a ring $R$ is Armendariz exactly when the map assigning to any right annihilator set $A \subseteq R$ the set of polynomials with coefficients in $A$ is a bijection onto the right annihilators of $R[x]$.

The pioneering paper [37] further proposes the study of rings with an analogue of the Armendariz condition defined with respect to power series rings. This proposal has been put into effect and extended: rings satisfying an Armendariz condition for generalized power series extensions, as well as rings satisfying such a condition for monoid rings, have been studied. The condition has also been defined and investigated for skew polynomial rings, for skew power series rings, and for skew monoid rings (see the beginning of Section 2 for details). But although these new classes of rings were all defined using generalizations or analogues of the Armendariz condition, the theory of each class was developed separately, which led to many papers with parallel results.

In this paper we propose a unified approach to all the above-mentioned classes of rings. The idea is to study the Armendariz condition defined for the skew generalized power series ring $R[[S, \omega]]$, where $R$ is a ring, $S$ is a strictly ordered monoid, and $\omega: S \rightarrow \operatorname{End}(R)$ is a monoid homomorphism (the definition of $R[[S, \omega]]$ will be stated in a moment). Since (skew) polynomial rings, (skew) power series rings, (skew) monoid rings, and generalized power series rings are particular cases of the $R[[S, \omega]]$ ring construction, the class of Armendariz rings as well as all the above-mentioned classes of Armendariz-like rings are subclasses of the new class of $(S, \omega)$-Armendariz rings. Hence any result on $(S, \omega)$-Armendariz rings has its counterpart in each of the subclasses, and these counterparts follow immediately from a single proof.

In this paper we extend to $(S, \omega)$-Armendariz rings many results known earlier for particular types of Armendariz-like rings. Nevertheless, we wish to emphasize that some of our results are new even for Armendariz rings; for example, we prove that left uniserial rings are Armendariz (Corollary 6.3). It is worth noting that Lee and Zhou [23] recently proposed an interesting unification of Armendariz rings and power-serieswise Armendariz rings (see Section 2 for a definition). Our approach to the unification of Armendariz-like rings in this paper is completely different from Lee and Zhou's, from the standpoint both of the ring extensions studied and of the structural properties obtained.

The paper is organized as follows. In Section 2 we introduce $(S, \omega)$-Armendariz rings. In Section 3 we give a lattice structure to the right annihilators of a ring and characterize $(S, \omega)$-Armendariz rings as those rings $R$ for which an analogue of the Hirano map is a lattice isomorphism from the right annihilators of $R$ to the right annihilators of $R[[S, \omega]]$. A detailed analysis of the map is rewarded with the removal of the Armendariz hypothesis from the main result of [19]. 
In Section 4 we study relationships between $(S, \omega)$-Armendariz rings and some important generalizations of commutative rings. For instance, we prove that every $(S, \omega)$-Armendariz ring is abelian (Proposition 4.9). Furthermore, we characterize when a skew generalized power series ring is reduced (Theorem 4.12) or semicommutative (Theorem 4.1), and we obtain partial characterizations for it to be reversible or 2-primal. We pose a natural open problem (Question 4.14) on reversible and semicommutative skew generalized power series rings. We also obtain some information on nilpotent elements of $(S, \omega)$-Armendariz rings and prove that the Köthe conjecture has a positive solution in the class of $S$-compatible $(S, \omega)$ Armendariz rings (Proposition 4.5).

In Section 5 we study the behaviour of the $(S, \omega)$-Armendariz condition under ring extensions. In particular, we extend to $(S, \omega)$-Armendariz rings Anderson and Camillo's result [1, Theorem 5] that for any ring $R$ and any integer $n \geq 2$, the factor ring $R[x] /\left(x^{n}\right)$ is Armendariz if and only if $R$ is reduced (Theorem 5.4).

In Section 6 we obtain criteria for a left or right uniserial ring to be $(S, \omega)$ Armendariz. As a consequence of the main result of this section (Proposition 6.1), we infer that every left or right uniserial ring is Armendariz (and furthermore it is Armendariz relative to any unique product monoid).

In the closing Section 7 we study the $(S, \omega)$-Armendariz condition in triangular matrix rings.

Throughout this paper, rings are associative, and they contain an identity element. We will write monoids multiplicatively unless otherwise indicated. If $R$ is a ring and $X$ is a nonempty subset of $R$, then the left (right) annihilator of $X$ in $R$ is denoted by $\operatorname{ann}_{\ell}^{R}(X)\left(\operatorname{ann}_{r}^{R}(X)\right)$. We will denote by $\operatorname{End}(R)$ the monoid of ring endomorphisms of $R$, and by $\operatorname{Aut}(R)$ the group of ring automorphisms of $R$. For later reference, we recall that a module $M$ is said to be uniserial if the submodule lattice of $M$ is totally ordered; a ring $R$ is said to be right (left) uniserial if the module $R_{R}\left({ }_{R} R\right)$ is uniserial.

In order to recall the skew generalized power series ring construction, we need some definitions. Let $(S, \leq)$ be a partially ordered set. Then $(S, \leq)$ is called artinian if every strictly decreasing sequence of elements of $S$ is finite, and $(S, \leq)$ is called narrow if every subset of pairwise order-incomparable elements of $S$ is finite. Thus, $(S, \leq)$ is artinian and narrow if and only if every nonempty subset of $S$ has at least one but only a finite number of minimal elements.

An ordered monoid is a pair $(S, \leq)$ consisting of a monoid $S$ and an order $\leq$ on $S$ such that for all $a, b, c \in S, a \leq b$ implies $c a \leq c b$ and $a c \leq b c$. An ordered monoid $(S, \leq)$ is said to be strictly ordered if for all $a, b, c \in S, a<b$ implies $c a<c b$ and $a c<b c$.

Let $R$ be a ring, $(S, \leq)$ a strictly ordered monoid, and $\omega: S \rightarrow \operatorname{End}(R)$ a monoid homomorphism. For $s \in S$, let $\omega_{s}$ denote the image of $s$ under $\omega$, that is, $\omega_{s}=\omega(s)$. Let $A$ be the set of all functions $f: S \rightarrow R$ such that the $\operatorname{support} \operatorname{supp}(f)=\{s \in S$ : $f(s) \neq 0\}$ is artinian and narrow. Then for any $s \in S$ and $f, g \in A$ the set

$$
\mathrm{X}_{s}(f, g)=\{(x, y) \in \operatorname{supp}(f) \times \operatorname{supp}(g): s=x y\}
$$


is finite. Thus one can define the product $f g: S \rightarrow R$ of $f, g \in A$ as follows:

$$
(f g)(s)=\sum_{(x, y) \in \mathrm{X}_{s}(f, g)} f(x) \cdot \omega_{x}(g(y))
$$

(by convention, a sum over the empty set is 0 ). With pointwise addition and multiplication as defined above, $A$ becomes a ring, called the ring of skew generalized power series with coefficients in $R$ and exponents in $S$ (see [31]), denoted by $R[[S, \omega, \leq]]$ (or by $R[[S, \omega]]$ when there is no ambiguity concerning the order $\leq$ ). The skew generalized power series construction embraces a wide range of classical ring-theoretic extensions, including skew polynomial rings, skew power series rings, skew Laurent polynomial rings, skew group rings, Mal'cev-Neumann Laurent series rings, and of course the 'untwisted' versions of all of these.

We will use the symbol 1 to denote the identity elements of the monoid $S$, the ring $R$, and the ring $R[[S, \omega]]$, as well as the trivial monoid homomorphism $1: S \rightarrow$ $\operatorname{End}(R)$ that sends every element of $S$ to the identity endomorphism. A subset $P \subseteq R$ will be called $S$-invariant if for every $s \in S$ it is $\omega_{s}$-invariant (that is, $\omega_{s}(P) \subseteq P$ ).

To each $r \in R$ and $s \in S$, we associate elements $\mathrm{C}_{r}, \mathrm{e}_{s} \in R[[S, \omega]]$ defined by

$$
\mathrm{c}_{r}(x)=\left\{\begin{array}{ll}
r & \text { if } x=1 \\
0 & \text { if } x \in S \backslash\{1\},
\end{array} \quad \mathrm{e}_{s}(x)= \begin{cases}1 & \text { if } x=s \\
0 & \text { if } x \in S \backslash\{s\} .\end{cases}\right.
$$

It is clear that $r \mapsto \mathrm{C}_{r}$ is a ring embedding of $R$ into $R[[S, \omega]]$ and $s \mapsto \mathrm{e}_{s}$ is a monoid embedding of $S$ into the multiplicative monoid of the ring $R[[S, \omega]]$, and $\mathrm{e}_{s} \mathrm{c}_{r}=\mathrm{c}_{\omega_{s}(r)} \mathrm{e}_{s}$. Moreover, for each nonempty subset $X$ of $R$ we put

$$
X[[S, \omega]]=\{f \in R[[S, \omega]]: f(s) \in X \cup\{0\} \text { for every } s \in S\},
$$

and for each nonempty subset $Y$ of $R[[S, \omega]]$ we put $C_{Y}=\{g(t): g \in Y, t \in S\}$.

\section{2. $(S, \omega)$-Armendariz rings}

A ring $R$ is called Armendariz if whenever polynomials $f(x)=a_{0}+a_{1} x+\cdots+$ $a_{m} x^{m}$ and $g(x)=b_{0}+b_{1} x+\cdots+b_{n} x^{n}$ in $R[x]$ satisfy $f(x) g(x)=0$, then $a_{i} b_{j}=0$ for each $i, j$. This definition was coined by Rege and Chhawchharia in [37] in recognition of Armendariz's proof in [3, Lemma 1] that reduced rings satisfy this condition. The Armendariz condition, and various derivatives described below, have been studied by numerous authors.

Given a ring $R$ and a ring endomorphism $\sigma: R \rightarrow R$, the skew polynomial ring $R[x ; \sigma]$ consists of polynomials in the indeterminate $x$ with coefficients from $R$, written on the left, where multiplication in $R[x ; \sigma]$ is defined by

$$
\left(\sum_{i} a_{i} x^{i}\right)\left(\sum_{j} b_{j} x^{j}\right)=\sum_{i, j} a_{i} \sigma^{i}\left(b_{j}\right) x^{i+j}
$$


Following Hong et al. [13], we say that a ring $R$ with an endomorphism $\sigma$ is $\sigma$-skew Armendariz if whenever polynomials $f(x)=a_{0}+a_{1} x+\cdots+a_{m} x^{m}$ and $g(x)=$ $b_{0}+b_{1} x+\cdots+b_{n} x^{n}$ in $R[x ; \sigma]$ satisfy $f(x) g(x)=0$ then $a_{i} \sigma^{i}\left(b_{j}\right)=0$ for all $i, j$. A stronger condition than Armendariz was studied by Kim et al. in [18]. A ring $R$ is said to be power-serieswise Armendariz if whenever power series $f(x)=\sum_{i=0}^{\infty} a_{i} x^{i}$ and $g(x)=\sum_{j=0}^{\infty} b_{j} x^{j}$ in $R[[x]]$ satisfy $f(x) g(x)=0$ then $a_{i} b_{j}=0$ for all $i, j$. In [25], Liu extended the Armendariz notion to monoid rings. If $R$ is a ring and $S$ is a monoid, then $R$ is called an Armendariz ring relative to $S$ if whenever elements $f=a_{1} s_{1}+a_{2} s_{2}+\cdots+a_{m} s_{m}$ and $g=b_{1} t_{1}+b_{2} t_{2}+\cdots+b_{n} t_{n}$ of the monoid ring $R[S]$ satisfy $f g=0$, then $a_{i} b_{j}=0$ for all $i, j$. In the case of commutative monoids, Liu generalized this definition in [24] to (untwisted) generalized power series rings as follows. If $R$ is a ring and $(S, \leq)$ is a commutative strictly ordered monoid, then $R$ is called $S$-Armendariz if whenever generalized power series $f, g \in R[[S, 1]]$ satisfy $f g=0$, then $f(s) g(t)=0$ for all $s, t \in S$.

We unify the above versions of Armendariz rings by introducing the following definition.

Definition 2.1. Let $R$ be a ring, $(S, \leq)$ a strictly ordered monoid, and $\omega: S \rightarrow$ $\operatorname{End}(R)$ a monoid homomorphism. We say that $R$ is $(S, \omega)$-Armendariz (or $(S, \omega, \leq)$ Armendariz to indicate the order $\leq$ ) if whenever $f g=0$ for $f, g \in R[[S, \omega]]$, then $f(s) \cdot \omega_{s}(g(t))=0$ for all $s, t \in S$.

If $S=\{1\}$ then every ring is $(S, \omega)$-Armendariz. In some of our results we will stipulate that $S \neq\{1\}$ to avoid trivialities.

EXAMPLE 2.2. Here are some special cases of $(S, \omega)$-Armendariz rings.

(i) Suppose $R$ is Armendariz, as in [37]. This is the special case where $S=\mathbb{N} \cup\{0\}$ under addition, with the trivial order, and $\omega$ is trivial.

(ii) Suppose $R$ is $\sigma$-skew Armendariz for some $\sigma \in \operatorname{End}(R)$, as in [13]. This is the special case where $S=\mathbb{N} \cup\{0\}$ under addition, with the trivial order, and $\omega$ is determined by $\omega(1)=\sigma$.

(iii) Suppose $R$ is power-serieswise Armendariz, as in [18]. This is the special case where $S=\mathbb{N} \cup\{0\}$ under addition, with its natural linear order, and $\omega$ is trivial.

(iv) Suppose $R$ is Armendariz relative to a monoid $S$, as in [25]. This is the special case where $S$ is given the trivial order, and $\omega$ is trivial.

(v) Suppose $R$ is $S$-Armendariz for some commutative, strictly ordered monoid $(S, \leq)$, as in [24]. This is the special case where $\omega$ is trivial (and $S$ satisfies the extra conditions just described).

We recall the definition of a compatible endomorphism from [2, Definition 2.1].

DEFINITION 2.3. An endomorphism $\sigma$ of a ring $R$ is called compatible if for all $a, b \in R$,

$$
a b=0 \Leftrightarrow a \sigma(b)=0 .
$$


Compatibility arises naturally in the study of $(S, \omega)$-Armendariz rings. To see why, suppose $R$ is a ring and $\sigma$ is an endomorphism of $R$. Then the skew power series ring $R[[x ; \sigma]]$ is a skew generalized power series ring for $S=\mathbb{N} \cup\{0\}$ with natural order $\leq$ and $\omega(n)=\sigma^{n}$. Notice that for elements $a$ and $b$ of an $(S, \omega)$-Armendariz ring $R$, if $a b=0$, then $a \sigma(b)=0$ (that is, 'half' of the definition of compatibility must hold). Indeed, define $f, g \in R[[x ; \sigma]]$ as follows:

$$
f=a-a x, \quad g=b+\sigma(b) x+\sigma^{2}(b) x^{2}+\sigma^{3}(b) x^{3}+\cdots .
$$

Then $f g=0$, and invoking the $(S, \omega)$-Armendariz condition for the constant coefficient of $f$ and the $x$-coefficient of $g$ yields $a \sigma(b)=0$.

We will also want to consider a condition on endomorphisms stronger than compatibility, namely the rigidity condition studied in [20]. For example, we will use this condition to characterize the $(S, \omega)$-Armendariz property in an appropriate setting in Theorem 4.12.

DEFINITION 2.4. An endomorphism $\sigma$ of a ring $R$ is called rigid if for every $a \in R$,

$$
a \sigma(a)=0 \Leftrightarrow a=0 .
$$

Let $R$ be a ring, $(S, \leq)$ a strictly ordered monoid and $\omega: S \rightarrow \operatorname{End}(R)$ a monoid homomorphism. We say that $R$ is $S$-compatible ( $S$-rigid) if $\omega_{s}$ is compatible (rigid) for every $s \in S$; to indicate the homomorphism $\omega$, we will sometimes say that $R$ is $(S, \omega)$-compatible $((S, \omega)$-rigid $)$.

Basic properties of rigid and compatible endomorphisms, proved by Hashemi and Moussavi in [11, Lemmas 2.1 and 2.2], are summarized here:

LEMMA 2.5. Let $\sigma$ be an endomorphism of a ring $R$. Then:

(i) if $\sigma$ is compatible, then $\sigma$ is injective;

(ii) $\sigma$ is compatible if and only if for all $a, b \in R, \sigma(a) b=0 \Leftrightarrow a b=0$;

(iii) the following conditions are equivalent:

(1) $\sigma$ is rigid;

(2) $\sigma$ is compatible and $R$ is reduced;

(3) for every $a \in R, \sigma(a) a=0$ implies that $a=0$.

It will be useful to establish criteria for transfer of the $(S, \omega)$-Armendariz condition from one ring to another. Let $R_{1}$ and $R_{2}$ be rings, $\left(S_{1}, \leq_{1}\right)$ and $\left(S_{2}, \leq_{2}\right)$ strictly ordered monoids, and let $v: S_{1} \rightarrow \operatorname{End}\left(R_{1}\right)$ and $\omega: S_{2} \rightarrow \operatorname{End}\left(R_{2}\right)$ be monoid homomorphisms. Let $\alpha: S_{1} \rightarrow S_{2}$ be a monoid monomorphism such that for any artinian and narrow subset $T$ of $S_{1}, \alpha(T)$ is an artinian and narrow subset of $S_{2}$, and let $\varphi: R_{1} \rightarrow R_{2}$ be a ring homomorphism such that for every $s \in S_{1}$ the following diagram is commutative.

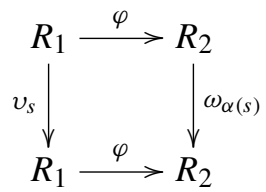


For $f \in R_{1}\left[\left[S_{1}, v, \leq_{1}\right]\right]$, let $\bar{f}: S_{2} \rightarrow R_{2}$ be the map defined as follows:

$$
\bar{f}(x)= \begin{cases}\varphi \circ f \circ \alpha^{-1}(x) & \text { if } x \in \alpha\left(S_{1}\right) \\ 0 & \text { otherwise }\end{cases}
$$

It is easy to see that $\operatorname{supp}(\bar{f}) \subseteq \alpha(\operatorname{supp}(f))$, and thus $\bar{f} \in R_{2}\left[\left[S_{2}, \omega, \leq_{2}\right]\right]$. Putting $\Phi(f)=\bar{f}$, we define a map $\Phi: R_{1}\left[\left[S_{1}, v, \leq_{1}\right]\right] \rightarrow R_{2}\left[\left[S_{2}, \omega, \leq_{2}\right]\right]$. Fixing all of this notation, we have the following two lemmas, the proofs of which we suppress.

LEMMA 2.6. The map $\Phi: R_{1}\left[\left[S_{1}, v, \leq_{1}\right]\right] \rightarrow R_{2}\left[\left[S_{2}, \omega, \leq_{2}\right]\right]$ is a ring homomorphism, and $\operatorname{ker} \Phi=(\operatorname{ker} \varphi)\left[\left[S_{1}, v\right]\right]$.

LEMMA 2.7. If $\varphi: R_{1} \rightarrow R_{2}$ is injective and $R_{2}$ is $\left(S_{2}, \omega\right)$-Armendariz, then $R_{1}$ is $\left(S_{1}, v\right)$-Armendariz.

The following proposition provides us with a method of constructing $(S, \omega)$ Armendariz rings. Recall that an ordered monoid $(S, \leq)$ is left naturally ordered if for all $s, t \in S, s \leq t$ implies that $t \in S s$ (see [33]).

PRoposition 2.8. Let $R$ be a domain, $(S, \leq)$ a strictly ordered monoid, and $\omega: S \rightarrow$ $\operatorname{End}(R)$ a monoid homomorphism. Assume that the order $\leq$ can be refined to a strict total order $\preccurlyeq$ such that the monoid $(S, \preccurlyeq)$ is left naturally ordered. Then $R$ is an $(S, \omega)$-Armendariz ring.

Proof. By Lemma 2.7 it suffices to show that $R$ is $(S, \omega, \preccurlyeq)$-Armendariz. Assume that $R$ is not an $(S, \omega, \preccurlyeq)$-Armendariz ring. Then there exist $f, g \in R[[S, \omega]]$ such that $f g=0$ but the set

$$
H=\left\{(s, t) \in S \times S: f(s) \cdot \omega_{s}(g(t)) \neq 0\right\}
$$

is nonempty. The sets $\operatorname{supp}(f)$ and $\operatorname{supp}(g)$ are well-ordered with respect to $\preccurlyeq$ and $H \subseteq \operatorname{supp}(f) \times \operatorname{supp}(g)$, so we can choose an element $\left(s_{0}, t_{0}\right) \in H$ minimal with respect to the lexicographic order $\preccurlyeq$ lex .

Suppose that there exists $(s, t) \in H \backslash\left\{\left(s_{0}, t_{0}\right)\right\}$ such that $s t=s_{0} t_{0}$. By the choice of $\left(s_{0}, t_{0}\right)$ we have $s_{0} \preccurlyeq s$. Since the order $\preccurlyeq$ is strict and total, and $s t=s_{0} t_{0}$, and $(s, t) \neq\left(s_{0}, t_{0}\right)$, it follows that $s_{0} \prec s$. Thus $t \prec t_{0}$, and consequently $\left(s_{0}, t\right) \prec_{\text {lex }}$ $\left(s_{0}, t_{0}\right)$. Hence the minimality of $\left(s_{0}, t_{0}\right)$ implies that $f\left(s_{0}\right) \cdot \omega_{s_{0}}(g(t))=0$, and since $R$ is a domain, we obtain $\omega_{s_{0}}(g(t))=0$. Furthermore, since $s_{0} \prec s$, there exists $z \in S$ such that $s=z s_{0}$, and thus $\omega_{s}(g(t))=\omega_{z}\left(\omega_{s_{0}}(g(t))\right)=0$, contradicting $(s, t) \in H$.

By the above, the only element $(s, t) \in H$ with $s t=s_{0} t_{0}$ is $(s, t)=\left(s_{0}, t_{0}\right)$. Therefore, since $\left(s_{0}, t_{0}\right) \in H$ and $f g=0$, we obtain $0 \neq f\left(s_{0}\right) \cdot \omega_{s_{0}}\left(g\left(t_{0}\right)\right)=$ $(f g)\left(s_{0} t_{0}\right)=0$, a contradiction.

Since the trivial order on the additive monoid $S=\mathbb{N} \cup\{0\}$ can be refined to the usual order $\leq$ and $S$ is naturally ordered by $\leq$, from Proposition 2.8 and Example 2.2(ii), we obtain the following result of Hong, Kim, and Kwak. 
COROllary 2.9 [13, Proposition 10]. If $R$ is a domain, then $R$ is $\sigma$-skew Armendariz for any endomorphism $\sigma$ of $R$.

\section{Characterizations of $(S, \omega)$-Armendariz rings via annihilators}

In this section we will present a characterization theorem for $(S, \omega)$-Armendariz rings in terms of one-sided annihilators. The result involves the sets $X[[S, \omega]]$ and $\mathrm{C}_{Y}$ defined in Section 1. Recall that if $(S, \leq)$ is a strictly ordered monoid, $R$ is a ring, $\omega: S \rightarrow \operatorname{End}(R)$ is a monoid homomorphism and $A=R[[S, \omega]]$, then for $\emptyset \neq X \subseteq R$,

$$
X[[S, \omega]]=\{f \in A: f(s) \in X \cup\{0\} \text { for every } s \in S\},
$$

and for $\emptyset \neq Y \subseteq A$,

$$
\mathrm{C}_{Y}=\{g(t): g \in Y, t \in S\}
$$

Note that

$$
X \cup\{0\}=\mathrm{C}_{X[[S, \omega]]} \quad \text { for any nonempty subset } X \text { of } R .
$$

Now, let $\mathcal{S}(R)$ (respectively $\mathcal{S}(A)$ ) be the set of nonempty subsets of $R$ (respectively $A)$. We obtain the following maps:

$$
\begin{array}{ll}
\alpha: \mathcal{S}(R) \rightarrow \mathcal{S}(A) & \text { defined by } \alpha(X)=X[[S, \omega]], \\
\beta: \mathcal{S}(A) \rightarrow \mathcal{S}(R) & \text { defined by } \beta(Y)=\mathrm{C}_{Y} .
\end{array}
$$

As we will see in Theorem 3.4, an $S$-compatible ring $R$ is $(S, \omega)$-Armendariz exactly when the following diagram is commutative:

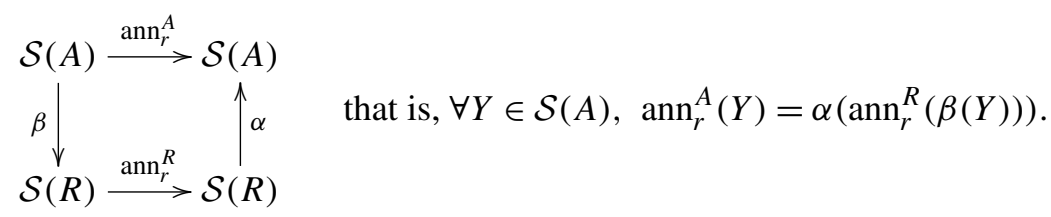

Other equivalent conditions involving annihilators also will be given in the theorem.

We start by characterizing $S$-compatible rings.

LEMMA 3.1. Let $R$ be a ring, $(S, \leq)$ a strictly ordered monoid, $\omega: S \rightarrow \operatorname{End}(R) a$ monoid homomorphism, and $A=R[[S, \omega]]$.

(1) The following conditions are equivalent:

(i) $R$ is $S$-compatible;

(ii) for any $a \in R$ and any nonempty subset $Y \subseteq A$,

$$
a \in \operatorname{ann}_{r}^{R}\left(C_{Y}\right) \Leftrightarrow \mathrm{C}_{a} \in \operatorname{ann}_{r}^{A}(Y) .
$$

(2) For any $a \in R$ and any nonempty subset $Y \subseteq A$,

$$
a \in \operatorname{ann}_{\ell}^{R}\left(C_{Y}\right) \Leftrightarrow \mathrm{C}_{a} \in \operatorname{ann}_{\ell}^{A}(Y) .
$$


Proof. (1) (i) $\Rightarrow$ (ii). Assume that $R$ is $S$-compatible. Then for any $a \in R$ and $f \in A$,

$$
\begin{aligned}
a \in \operatorname{ann}_{r}^{R}\left(\mathrm{C}_{\{f\}}\right) \Leftrightarrow \mathrm{C}_{\{f\}} a=\{0\} & \Leftrightarrow \forall s \in S, \quad f(s) a=0 \\
& \Leftrightarrow \forall s \in S, \quad f(s) \cdot \omega_{s}(a)=0 \\
& \Leftrightarrow \forall s \in S, \quad\left(f \mathrm{C}_{a}\right)(s)=0 \\
& \Leftrightarrow f \mathrm{C}_{a}=0 \\
& \Leftrightarrow \mathrm{C}_{a} \in \operatorname{ann}_{r}^{A}(f),
\end{aligned}
$$

and (ii) follows.

(ii) $\Rightarrow$ (i). Let $a, b \in R$ and $s \in S$. Then using (ii) we obtain

$$
\begin{aligned}
a b=0 \Leftrightarrow b \in \operatorname{ann}_{r}^{R}(\{a, 0\}) \Leftrightarrow b \in \operatorname{ann}_{r}^{R}\left(\mathrm{C}_{\left\{\mathrm{c}_{a} \mathrm{e}_{s}\right\}}\right) & \Leftrightarrow \mathrm{c}_{b} \in \operatorname{ann}_{r}^{A}\left(\mathrm{c}_{a} \mathrm{e}_{s}\right) \\
& \Leftrightarrow \mathrm{c}_{a} \mathrm{e}_{s} \mathrm{c}_{b}=0 \\
& \Leftrightarrow a \cdot \omega_{s}(b)=0 .
\end{aligned}
$$

Thus, $R$ is $S$-compatible.

(2) This can be proved similarly to (i) $\Rightarrow$ (ii) of (1) (we do not need any assumption about $S$-compatibility in this case).

LEMMA 3.2. Let $R$ be a ring, $(S, \leq)$ a strictly ordered monoid, $\omega: S \rightarrow \operatorname{End}(R) a$ monoid homomorphism, and $A=R[[S, \omega]]$. If $R$ is $S$-compatible, then:

(i) $\quad$ for any nonempty subset $X \subseteq R, \operatorname{ann}_{r}^{R}(X)[[S, \omega]]=\operatorname{ann}_{r}^{A}(X[[S, \omega]])$;

(ii) for any nonempty subset $X \subseteq R, \operatorname{ann}_{r}^{R}(X)$ is an ideal of $R$ if and only if $\operatorname{ann}_{r}^{R}(X)[[S, \omega]]$ is an ideal of $A ;$

$\left(\mathrm{i}^{\prime}\right)-\left(\mathrm{ii}^{\prime}\right)$ the analogues of $(\mathrm{i})-(\mathrm{ii})$ for left annihilators.

Proof. (i) The inclusion $\operatorname{ann}_{r}^{R}(X)[[S, \omega]] \subseteq \operatorname{ann}_{r}^{A}(X[[S, \omega]])$ is clear from $S$-compatibility. To prove the opposite inclusion, consider any $f \in \operatorname{ann}_{r}^{A}(X[[S, \omega]])$, $s \in S$, and $x \in X$. Since $\mathrm{c}_{x} \in X[[S, \omega]]$, we have $\mathrm{c}_{x} f=0$ and thus $x f(s)=$ $\left(\mathrm{C}_{x} f\right)(s)=0$, which shows that $f \in \operatorname{ann}_{r}^{R}(X)[[S, \omega]]$.

(ii) Assume that $\operatorname{ann}_{r}^{R}(X)$ is an ideal of $R$. By (i), $\operatorname{ann}_{r}^{R}(X)[[S, \omega]]$ is a right ideal of $A$. Choose any $f \in A$ and $g \in \operatorname{ann}_{r}^{R}(X)[[S, \omega]]$. Let $s, t \in S$. Then $g(t) \in \operatorname{ann}_{r}^{R}(X)$, and the $S$-compatibility of $R$ yields $\omega_{s}(g(t)) \in \operatorname{ann}_{r}^{R}(X)$. By hypothesis $\operatorname{ann}_{r}^{R}(X)$ is an ideal of $R$; thus, $f(s) \cdot \omega_{s}(g(t)) \in \operatorname{ann}_{r}^{R}(X)$. Hence for any $z \in S$ we have $(f g)(z) \in \operatorname{ann}_{r}^{R}(X)$, which shows that $f g \in \operatorname{ann}_{r}^{R}(X)[[S, \omega]]$.

Conversely, assume that $\operatorname{ann}_{r}^{R}(X)[[S, \omega]]$ is an ideal of $A$. Then for any $a \in R$ and $r \in \operatorname{ann}_{r}^{R}(X)$ we have $\mathrm{C}_{a r}=\mathrm{C}_{a} \mathrm{c}_{r} \in A \cdot \operatorname{ann}_{r}^{R}(X)[[S, \omega]] \subseteq \operatorname{ann}_{r}^{R}(X)[[S, \omega]]$, and thus ar $=\mathrm{C}_{a r}(1) \in \operatorname{ann}_{r}^{R}(X)$. Hence $\operatorname{ann}_{r}^{R}(X)$ is an ideal of $R$.

$\left(\mathrm{i}^{\prime}\right)-\left(\mathrm{ii}^{\prime}\right)$ The proofs are analogous.

Following Camillo et al. [6], we say that a ring $R$ is right Ikeda-Nakayama if

$$
\operatorname{ann}_{\ell}^{R}(I \cap J)=\operatorname{ann}_{\ell}^{R}(I)+\operatorname{ann}_{\ell}^{R}(J)
$$


for all right ideals $I, J$ of $R$. As observed in [6], the right Ikeda-Nakayama condition fits nicely into the following ring-theoretic implication diagram.

right self-injective

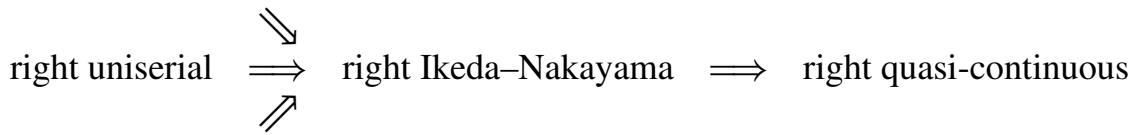

right Ore domain

PROPOSITION 3.3. Let $R$ be a ring, $(S, \leq)$ a strictly ordered monoid, and $\omega: S \rightarrow$ $\operatorname{End}(R)$ a monoid homomorphism. Assume that $R$ is $S$-compatible. If $R[[S, \omega]]$ is a right Ikeda-Nakayama ring, then so is $R$.

PROOF. Let $I$ and $J$ be right ideals of $R$. Trivially $\operatorname{ann}_{\ell}^{R}(I)+\operatorname{ann}_{\ell}^{R}(J) \subseteq \operatorname{ann}_{\ell}^{R}(I \cap$ $J)$. Consider any $a \in \operatorname{ann}_{\ell}^{R}(I \cap J)$, and set $A=R[[S, \omega]]$. Since $I[[S, \omega]]$ and $J[[S, \omega]]$ are right ideals of $A$, by the right Ikeda-Nakayama hypothesis and Lemma 3.2(i') we may write

$$
\begin{aligned}
\mathrm{C}_{a} \in \operatorname{ann}_{\ell}^{A}(I[[S, \omega]] \cap J[[S, \omega]]) & =\operatorname{ann}_{\ell}^{A}(I[[S, \omega]])+\operatorname{ann}_{\ell}^{A}(J[[S, \omega]]) \\
& =\operatorname{ann}_{\ell}^{R}(I)[[S, \omega]]+\operatorname{ann}_{\ell}^{R}(J)[[S, \omega]] .
\end{aligned}
$$

Hence $a \in \operatorname{ann}_{\ell}^{R}(I)+\operatorname{ann}_{\ell}^{R}(J)$.

In light of Example 2.2(i), Proposition 3.3 recovers the main result of [19] (along with its power-series analogue), showing furthermore that in that result the assumption that $R$ is Armendariz is superfluous.

We are now ready to characterize $(S, \omega)$-Armendariz rings among $S$-compatible rings as those for which there exists a specific bijection between the sets of right (equivalently, left) annihilator ideals of $R$ and of $R[[S, \omega]]$, generalizing annihilator characterizations of various classes of Armendariz-like rings given in [12, Proposition 3.1], [12, Proposition 3.4], and [18, Proposition 2.6].

To state the result we introduce the following notation. For a ring $R$ we put

$$
\begin{aligned}
& \mathcal{L}_{\mathrm{ann}_{r}}(R)=\left\{\operatorname{ann}_{r}^{R}(X): X \text { is a nonempty subset of } R\right\}, \\
& \mathcal{L}_{\mathrm{ann}_{\ell}}(R)=\left\{\operatorname{ann}_{\ell}^{R}(X): X \text { is a nonempty subset of } R\right\} .
\end{aligned}
$$

Ordered by inclusion, $\mathcal{L}_{\mathrm{ann}_{r}}(R)$ has the following lattice structure. Given $\mathfrak{A}, \mathfrak{B} \in$ $\mathcal{L}_{\mathrm{ann}_{r}}(R)$, the meet and join are defined by

$$
\mathfrak{A} \wedge \mathfrak{B}=\mathfrak{A} \cap \mathfrak{B} \quad \text { and } \quad \mathfrak{A} \vee \mathfrak{B}=\operatorname{ann}_{r}^{R}\left(\operatorname{ann}_{\ell}^{R}(\mathfrak{A} \cup \mathfrak{B})\right) .
$$

Notice that $\operatorname{ann}_{\ell}^{R}(\mathfrak{A} \cup \mathfrak{B})=\operatorname{ann}_{\ell}^{R}(\mathfrak{A}+\mathfrak{B})$, and thus $\mathfrak{A} \vee \mathfrak{B}=\operatorname{ann}_{r}^{R}\left(\operatorname{ann}_{\ell}^{R}(\mathfrak{A}+\mathfrak{B})\right)$. The lattice structure on $\mathcal{L}_{\mathrm{ann}_{\ell}}(R)$ is analogous. 
THEOREM 3.4. Let $R$ be a ring, $(S, \leq)$ a strictly ordered monoid, $\omega: S \rightarrow \operatorname{End}(R)$ a monoid homomorphism, and let $A=R[[S, \omega]]$. Assume that $R$ is $S$-compatible. Define

$$
\begin{aligned}
\varphi: & \mathcal{L}_{\mathrm{ann}_{r}}(R) \rightarrow \mathcal{L}_{\mathrm{ann}_{r}}(A) \text { given by } \operatorname{ann}_{r}^{R}(X) \mapsto \operatorname{ann}_{r}^{A}(X[[S, \omega]]) ; \\
\varphi^{\prime}: & \mathcal{L}_{\mathrm{ann}_{\ell}}(R) \rightarrow \mathcal{L}_{\mathrm{ann}_{\ell}}(A) \text { given by } \operatorname{ann}_{\ell}^{R}(X) \mapsto \operatorname{ann}_{\ell}^{A}(X[[S, \omega]]) ; \\
& \psi: \mathcal{L}_{\mathrm{ann}_{r}}(A) \rightarrow \mathcal{L}_{\mathrm{ann}_{r}}(R) \text { given by } \operatorname{ann}_{r}^{A}(Y) \mapsto \operatorname{ann}_{r}^{R}\left(C_{Y}\right) ; \\
& \psi^{\prime}: \mathcal{L}_{\mathrm{ann}_{\ell}}(A) \rightarrow \mathcal{L}_{\mathrm{ann}_{\ell}}(R) \text { given by } \operatorname{ann}_{\ell}^{A}(Y) \mapsto \operatorname{ann}_{\ell}^{R}\left(C_{Y}\right) .
\end{aligned}
$$

Then:

(1) (a) the map $\varphi$ is a lattice monomorphism;

$\left(\mathrm{a}^{\prime}\right)$ the map $\varphi^{\prime}$ is a lattice monomorphism;

(b) the map $\psi$ is a poset epimorphism;

$\left(\mathrm{b}^{\prime}\right)$ the map $\psi^{\prime}$ is a poset epimorphism.

(2) The following conditions are equivalent:

(i) $R$ is $(S, \omega)$-Armendariz;

(ii) for every nonempty subset $Y$ of $A, \mathrm{C}_{\mathrm{ann}_{r}^{A}(Y)}=\operatorname{ann}_{r}^{R}\left(\mathrm{C}_{Y}\right)$;

(ii') for every nonempty subset $Y$ of $A, \mathrm{C}_{\mathrm{ann}_{\ell}^{A}(Y)}=\operatorname{ann}_{\ell}^{R}\left(\mathrm{C}_{Y}\right)$;

(iii) for every nonempty subset $Y$ of $A, \operatorname{ann}_{r}^{A}(Y)=\operatorname{ann}_{r}^{R}\left(C_{Y}\right)[[S, \omega]]$;

(iii') for every nonempty subset $Y$ of $A, \operatorname{ann}_{\ell}^{A}(Y)=\operatorname{ann}_{\ell}^{R}\left(C_{Y}\right)[[S, \omega]]$;

(iv) the map $\varphi$ is surjective;

(iv') the map $\varphi^{\prime}$ is surjective;

(v) the map $\psi$ is injective;

$\left(\mathrm{v}^{\prime}\right)$ the map $\psi^{\prime}$ is injective;

(vi) the map $\varphi$ is a lattice isomorphism;

(vi') the map $\varphi^{\prime}$ is a lattice isomorphism;

(vii) the map $\psi$ is a lattice isomorphism;

(vii') the map $\psi^{\prime}$ is a lattice isomorphism.

Proof. (1) By Lemma 3.2(i),

$$
\varphi(\mathfrak{C})=\mathfrak{C}[[S, \omega]] \quad \text { for any } \mathfrak{C} \in \mathcal{L}_{\text {ann }_{r}}(R) ;
$$

thus, $\varphi$ is injective, and for any $\mathfrak{A}, \mathfrak{B} \in \mathcal{L}_{\mathrm{ann}_{r}}(R)$ we have $\varphi(\mathfrak{A} \wedge \mathfrak{B})=\varphi(\mathfrak{A}) \wedge \varphi(\mathfrak{B})$. Applying Lemma 3.2(i') and equation (3.2), we obtain

$$
\begin{aligned}
\varphi(\mathfrak{A} \vee \mathfrak{B}) & =\operatorname{ann}_{r}^{A}\left(\operatorname{ann}_{\ell}^{R}(\mathfrak{A}+\mathfrak{B})[[S, \omega]]\right) \\
& =\operatorname{ann}_{r}^{A}\left(\operatorname{ann}_{\ell}^{A}((\mathfrak{A}+\mathfrak{B})[[S, \omega]])\right) \\
& =\operatorname{ann}_{r}^{A}\left(\operatorname{ann}_{\ell}^{A}(\mathfrak{A}[[S, \omega]]+\mathfrak{B}[[S, \omega]])\right) \\
& =\varphi(\mathfrak{A}) \vee \varphi(\mathfrak{B}) .
\end{aligned}
$$

This proves (a). The proof of $\left(a^{\prime}\right)$ is similar. By Lemma 3.1 the maps $\psi$ and $\psi^{\prime}$ are isotone, and Equation (3.1) shows they are surjective. This proves (b) and ( $\left.b^{\prime}\right)$. 
(2) (i) $\Rightarrow$ (ii). To show that $\mathrm{C}_{\mathrm{ann}_{r}^{A}(Y)} \subseteq \operatorname{ann}_{r}^{R}\left(\mathrm{C}_{Y}\right)$, consider any $a \in \mathrm{C}_{\mathrm{ann}_{r}^{A}(Y)}$ and $b \in \mathrm{C}_{Y}$. Then $a=g(t)$ and $b=f(s)$ for some $g \in \operatorname{ann}_{r}^{A}(Y), f \in Y$ and $s, t \in S$. Since $R$ is $(S, \omega)$-Armendariz and $S$-compatible, $f g=0$ implies $0=f(s) g(t)=b a$, as desired. To show that $\operatorname{ann}_{r}^{R}\left(\mathrm{C}_{Y}\right) \subseteq \mathrm{C}_{\text {ann }}^{A}(Y)$, consider any $a \in \operatorname{ann}_{r}^{R}\left(\mathrm{C}_{Y}\right)$. By Lemma 3.1(1) we have $\mathrm{c}_{a} \in \operatorname{ann}_{r}^{A}(Y)$, and thus $a=\mathrm{c}_{a}(1) \in \mathrm{C}_{\operatorname{ann}_{r}^{A}(Y)}$.

(ii) $\Rightarrow$ (iii). Given $f \in \operatorname{ann}_{r}^{A}(Y)$, from (ii) we obtain $f(s) \in \mathrm{C}_{\mathrm{ann}_{r}^{A}(Y)}=$ $\operatorname{ann}_{r}^{R}\left(\mathrm{C}_{Y}\right)$ for every $s \in S$. Therefore $f \in \operatorname{ann}_{r}^{R}\left(\mathrm{C}_{Y}\right)[[S, \omega]]$, which proves that $\operatorname{ann}_{r}^{A}(Y) \subseteq \operatorname{ann}_{r}^{R}\left(C_{Y}\right)[[S, \omega]]$. To prove the opposite inclusion, consider any $g \in$ $\operatorname{ann}_{r}^{R}\left(\mathrm{C}_{Y}\right)[[S, \omega]]$ and $f \in Y$. For any $s, t \in S$ we have $f(s) \in \mathrm{C}_{Y}$ and $g(t) \in$ $\operatorname{ann}_{r}^{R}\left(\mathrm{C}_{Y}\right)$, and thus $f(s) g(t)=0$. Since $R$ is $S$-compatible, $f(s) \cdot \omega_{s}(g(t))=0$. Hence $f g=0$, so $g \in \operatorname{ann}_{r}^{A}(Y)$, as desired.

(iii) $\Rightarrow$ (iv). Surjectivity of $\varphi$ is immediate from (iii) and Lemma 3.2(i).

(iv) $\Rightarrow$ (vi). This follows by (1)(a).

(vi) $\Rightarrow$ (vii). By Equation (3.1), the map $\psi \circ \varphi$ is the identity on $\mathcal{L}_{\mathrm{ann}_{r}}(R)$. Thus, if $\varphi$ is a lattice isomorphism, so is $\psi$.

(vii) $\Rightarrow$ (v). This is obvious.

(v) $\Rightarrow$ (i). Let $f, g \in A$ be such that $f g=0$. From Equation (3.1) we obtain

$$
\operatorname{ann}_{r}^{R}\left(\mathrm{C}_{\{f\}}\right)=\operatorname{ann}_{r}^{R}\left(\mathrm{C}_{\mathrm{C}_{\{f\}}[[S, \omega]]}\right) \Rightarrow \psi\left(\operatorname{ann}_{r}^{A}(f)\right)=\psi\left(\operatorname{ann}_{r}^{A}\left(\mathrm{C}_{\{f\}}[[S, \omega]]\right)\right) .
$$

Now (v) implies that $\operatorname{ann}_{r}^{A}(f)=\operatorname{ann}_{r}^{A}\left(\mathrm{C}_{\{f\}}[[S, \omega]]\right)$, whence $g \in \operatorname{ann}_{r}^{A}\left(\mathrm{C}_{\{f\}}[[S, \omega]]\right)$. So $\mathrm{C}_{f(s)} g=0$ for every $s \in S$. Therefore, for every $t \in S$, we have $f(s) g(t)=$ $\left(\mathrm{C}_{f(s)} g\right)(t)=0$, and thus $f(s) \cdot \omega_{s}(g(t))=0$ by $S$-compatibility of $R$, which proves that $R$ is $(S, \omega)$-Armendariz.

The implications (i) $\Rightarrow\left(\mathrm{ii}^{\prime}\right) \Rightarrow\left(\mathrm{iii}^{\prime}\right) \Rightarrow\left(\mathrm{iv}^{\prime}\right) \Rightarrow\left(\mathrm{vi}^{\prime}\right) \Rightarrow\left(\mathrm{vii}^{\prime}\right) \Rightarrow\left(\mathrm{v}^{\prime}\right) \Rightarrow$ (i) follow symmetrically.

REMARK 3.5. The curious asymmetry between conditions $(1)\left((a),\left(a^{\prime}\right)\right)$ and (1)((b), $\left.\left(b^{\prime}\right)\right)$ of Theorem 3.4 is unavoidable: in general, $\psi$ is not a morphism in the category of lattices. Obviously, whenever $\psi: L_{1} \rightarrow L_{2}$ is a surjective map between lattices $L_{1}$ and $L_{2}$ with the property that $a \leq b$ if and only if $\psi(a) \leq \psi(b)$ for all $a, b \in L_{1}$, then $\psi$ is a lattice epimorphism. But this is not, in general, the situation in the context of Theorem 3.4, as the following example shows.

EXAmple 3.6. Put $B=\mathbb{Z} / 4 \mathbb{Z}$, and let $R=B \oplus B$ as an additive group, with multiplication in $R$ defined by

$$
\left(m_{1}, n_{1}\right)\left(m_{2}, n_{2}\right)=\left(m_{1} m_{2}, m_{1} n_{2}+m_{2} n_{1}\right) .
$$

Then $R$ is a ring. Let $A=R[[x]]$ be the ring of power series over $R$. Thus, $A \cong R[[S, \omega]]$ for the additive monoid $S=\mathbb{N} \cup\{0\}$ with the usual order and trivial $\omega$; obviously $R$ is $S$-compatible.

Let $f, g \in A$ be given by

$$
f=(2,0)+(2,1) x, \quad g=(2,1)+(2,0) x .
$$


Let $\mathfrak{A}, \mathfrak{B} \in \mathcal{L}_{\mathrm{ann}_{r}}(A)$ be defined by

$$
\mathfrak{A}=\operatorname{ann}_{r}^{A}(f), \quad \mathfrak{B}=\operatorname{ann}_{r}^{A}(g) .
$$

An easy calculation shows that $\mathcal{L}_{\mathrm{ann}_{r}}(R)$ is the following lattice:

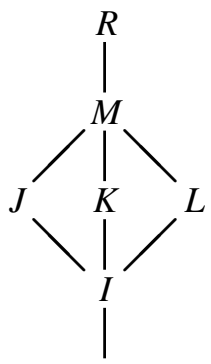

(0)

where

$$
M=\operatorname{ann}_{r}^{R}((0,2)), \quad J=\operatorname{ann}_{r}^{R}((0,1)), \quad K=\operatorname{ann}_{r}^{R}((2,0)), \quad L=\operatorname{ann}_{r}^{R}((2,1)),
$$

and $I=\{(0,0),(0,2)\}=\operatorname{ann}_{r}^{R}(M)$.

Since $\mathrm{C}_{\{f\}}=\mathrm{C}_{\{g\}}=\{(2,0),(2,1)\}$,

$$
\psi(\mathfrak{A})=\psi(\mathfrak{B})=\operatorname{ann}_{r}^{R}(\{(2,0),(2,1)\})=I \Rightarrow \psi(\mathfrak{A}) \vee \psi(\mathfrak{B})=I .
$$

Because $M$ is the maximal ideal of the local ring $R$, we have $\operatorname{ann}_{r}^{A}(f) \cup \operatorname{ann}_{r}^{A}(g) \subseteq$ $M[[x]]$; therefore, $I[[x]] \subseteq \operatorname{ann}_{\ell}^{A}\left(\operatorname{ann}_{r}^{A}(f) \cup \operatorname{ann}_{r}^{A}(g)\right)$. On the other hand, if $h(x)=$ $\sum_{n=0}^{\infty} \beta_{n} x^{n}$ is any nonzero element of $\operatorname{ann}_{\ell}^{A}\left(\operatorname{ann}_{r}^{A}(f) \cup \operatorname{ann}_{r}^{A}(g)\right)$, then since $h(x)$ annihilates both of the elements

$$
(2,0)+(2,1) x+(2,0) x^{2}+(2,1) x^{3}+(2,0) x^{4}+(2,1) x^{5}+\cdots \in \operatorname{ann}_{r}^{A}(f)
$$

and

$$
(2,1)+(2,0) x+(2,1) x^{2}+(2,0) x^{3}+(2,1) x^{4}+(2,0) x^{5}+\cdots \in \operatorname{ann}_{r}^{A}(g),
$$

a quick induction shows that for every $m \geq 0, \beta_{m} \in K \cap L=I$ and $\sum_{n=m+1}^{\infty} \beta_{n} x^{n} \in$ $\operatorname{ann}_{\ell}^{A}\left(\operatorname{ann}_{r}^{A}(f) \cup \operatorname{ann}_{r}^{A}(g)\right)$. Thus, $\operatorname{ann}_{\ell}^{A}\left(\operatorname{ann}_{r}^{A}(f) \cup \operatorname{ann}_{r}^{A}(g)\right) \subseteq I[[x]]$. This proves that

$$
\operatorname{ann}_{\ell}^{A}\left(\operatorname{ann}_{r}^{A}(f) \cup \operatorname{ann}_{r}^{A}(g)\right)=I[[x]],
$$

and therefore

$$
\psi(\mathfrak{A} \vee \mathfrak{B})=\operatorname{ann}_{r}^{R}\left(\mathrm{C}_{\operatorname{ann}_{\ell}^{A}\left(\operatorname{ann}_{r}^{A}(f) \cup \operatorname{ann}_{r}^{A}(g)\right)}\right)=\operatorname{ann}_{r}^{R}\left(\mathrm{C}_{I[[x]]}\right)=\operatorname{ann}_{r}^{R}(I)=M .
$$

Equations (3.3) and (3.4) show that $\psi(\mathfrak{A}) \vee \psi(\mathfrak{B})<\psi(\mathfrak{A} \vee \mathfrak{B})$. We conclude that in this case the poset epimorphism $\psi$ is not a lattice morphism (see Theorem 3.4(1)(b)).

As a consequence of Theorem 3.4, we obtain the following generalization of [12, Corollary 3.3], which was provided to counterpoint Kerr's example of a polynomial ring over a Goldie ring that is not a Goldie ring. 
COROLlary 3.7. Let $R$ be a ring, $(S, \leq)$ a strictly ordered monoid, and $\omega: S \rightarrow$ $\operatorname{End}(R)$ a monoid homomorphism. Suppose that $R$ is $S$-compatible and $(S, \omega)$ Armendariz. Then $R$ satisfies the ascending chain condition on right annihilators if and only if $R[[S, \omega]]$ satisfies the ascending chain condition on right annihilators.

\section{The $(S, \omega)$-Armendariz condition and generalizations of commutativity}

In this section we will obtain criteria for skew generalized power series rings to satisfy various conditions on noncommutative rings that generalize commutativity. A ring is called reduced if it contains no nonzero nilpotent elements. A ring $R$ is called reversible if for all $a, b \in R$ we have $a b=0$ if and only if $b a=0$. A ring $R$ is called semicommutative if for all $a, b \in R$ we have $a b=0 \Rightarrow a R b=\{0\}$. A ring is called abelian if every idempotent element is central. A ring is called 2-primal if its prime radical contains every nilpotent element of the ring. There is a substantial literature on these conditions, a survey of some of which can be found in [28]. The conditions have the following relationships, where the bottom left condition is defined with respect to any nontrivial strictly ordered monoid $(S, \leq)$ :

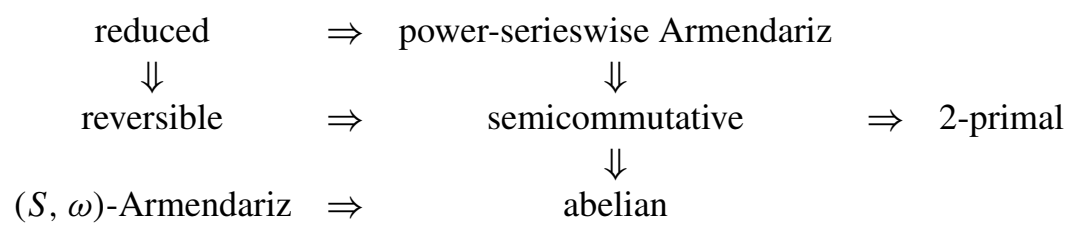

The implication 'reduced $\Rightarrow$ power-serieswise Armendariz', originally established in [18, Lemma 2.3(1)], is generalized in [29, Proposition 3.6] (see [29, Corollary 3.8(iii)]). The implication ' $(S, \omega)$-Armendariz $\Rightarrow$ abelian' follows from Proposition 4.9(ii) below. For 'power-serieswise Armendariz $\Rightarrow$ semicommutative', see [18, Lemma 2.3(2)]. The remaining implications are well known (see [28] and sources cited).

In the above diagram, the six conditions reduced, power-serieswise Armendariz, reversible, semicommutative, 2-primal, and abelian are equivalent for von Neumann regular rings. Thus, the characterizations of these conditions in skew generalized power series rings given below might be compared with the criteria obtained in [31] for a skew generalized power series ring to be von Neumann regular.

We first examine semicommutativity of skew generalized power series rings. Directly from Lemma 3.2 and Theorem 3.4 we obtain the following theorem.

THEOREM 4.1. Let $R$ be a ring, $(S, \leq)$ a strictly ordered monoid, and $\omega: S \rightarrow$ $\operatorname{End}(R)$ a monoid homomorphism. Assume that $R$ is $S$-compatible and $(S, \omega)$ Armendariz. Then $R[[S, \omega]]$ is semicommutative if and only if $R$ is.

Combining Theorem 4.1 and Example 2.2(i) recovers [37, Proposition 4.6].

In order to obtain criteria for $R$ and $R[[S, \omega]]$ to be semicommutative, we first derive some necessary conditions for a ring to be $(S, \omega)$-Armendariz. 
Recall that a monoid $S$ is cancellative if for all $s, t, z \in S, s \neq t$ implies $s z \neq t z$ and $z s \neq z t$. A monoid $S$ is aperiodic if for any $s \in S \backslash\{1\}$ and $m, n \in \mathbb{N}$ with $m \neq n$ we have $s^{m} \neq s^{n}$.

LEMMA 4.2. Let $R$ be a ring, $(S, \leq)$ a strictly ordered monoid, and $\omega: S \rightarrow \operatorname{End}(R)$ a monoid homomorphism. If $R$ is $(S, \omega)$-Armendariz, then:

(i) $S$ is cancellative;

(ii) $S$ is aperiodic;

(iii) if $x \in R$ and $s \in S \backslash\{1\}$ satisfy $x \cdot \omega_{s}(x) \cdot \omega_{s^{2}}(x) \cdots \omega_{s^{n}}(x)=0$ for some $n \in \mathbb{N}$, then for all $a, b \in R$ such that $a b=0$, we have $a \cdot x \cdot \omega_{s}(b)=0$.

PROOF. (i) Let $s, t \in S$ be such that $s \neq t$. Suppose that there exists $z \in S$ such that $s z=t z$. Then in $R[[S, \omega]]$ we have $\left(\mathrm{e}_{s}-\mathrm{e}_{t}\right) \mathrm{e}_{z}=0$, and since $R$ is $(S, \omega)$-Armendariz, it follows that $0=\left(\mathrm{e}_{s}-\mathrm{e}_{t}\right)(s) \cdot \omega_{s}\left(\mathrm{e}_{z}(z)\right)=1 \cdot \omega_{s}(1)=1$, a contradiction. Similarly, one can show that $s \neq t$ implies $z s \neq z t$.

(ii) Suppose that $S$ is not aperiodic. Applying (i), we deduce that there exists $s \in S \backslash\{1\}$ such that $s^{n}=1$ for some $n \in \mathbb{N}$. We can assume that $s^{i} \neq 1$ for each $i \in$ $\{1,2, \ldots, n-1\}$. Since $\left(1-\mathrm{e}_{s}\right)\left(1+\mathrm{e}_{s}+\mathrm{e}_{s^{2}}+\cdots+\mathrm{e}_{s^{n-1}}\right)=0$ and $R$ is $(S, \omega)$ Armendariz, we obtain

$$
0=\left(1-\mathrm{e}_{s}\right)(1) \cdot\left(1+\mathrm{e}_{s}+\mathrm{e}_{s^{2}}+\cdots+\mathrm{e}_{s^{n-1}}\right)(1)=1 \cdot 1=1,
$$

a contradiction.

(iii) Set $f=\mathrm{c}_{x} \mathrm{e}_{s} \in R[[S, \omega]]$. Since $x \cdot \omega_{s}(x) \cdot \omega_{s^{2}}(x) \cdots \omega_{s^{n}}(x)=0$, it follows that $f^{n+1}=0$, and thus

$$
\mathrm{c}_{a}(1-f)\left(1+f+f^{2}+\cdots+f^{n}\right) \mathrm{c}_{b}=\mathrm{c}_{a} \mathrm{c}_{b}=0 .
$$

Since $R$ is $(S, \omega)$-Armendariz, and $S$ is aperiodic by (ii), we obtain

$$
0=\left[\mathrm{c}_{a}(1-f)\right](1) \cdot\left[\left(1+f+f^{2}+\cdots+f^{n}\right) \mathrm{c}_{b}\right](s)=a \cdot x \cdot \omega_{s}(b) .
$$

PROposition 4.3. Let $R$ be a ring, $(S, \leq)$ a strictly ordered monoid, and $\omega: S \rightarrow$ $\operatorname{End}(R)$ a monoid homomorphism. If $R$ is $S$-compatible and $(S, \omega)$-Armendariz, and there exists $s \in S \backslash\{1\}$ such that $s^{m} \leq s^{n}$ for some positive integers $m<n$, then $R$ and $R[[S, \omega]]$ are semicommutative.

PROOF. By Theorem 4.1, to prove the result it suffices to show that $R$ is semicommutative. By [31, Lemma 4], the set $\left\{1, s, s^{2}, \ldots\right\}$ is artinian and narrow, and by Lemma 4.2(ii), for all $i, j \in \mathbb{N} \cup\{0\}$ with $i \neq j$ we have $s^{i} \neq s^{j}$. Take any $r \in R$ and define $f \in R[[S, \omega]]$ by setting

$$
f(1)=1, \quad f\left(s^{n}\right)=r \cdot \omega_{s}(r) \cdot \omega_{s^{2}}(r) \cdots \omega_{s^{n-1}}(r) \text { for every } n \in \mathbb{N},
$$

and $f(x)=0$ for every $x \in S \backslash\left\{1, s, s^{2}, \ldots\right\}$. It is easy to see that $\left(1-\mathrm{c}_{r} \mathrm{e}_{s}\right) f=1$. Therefore, for any $a, b \in R$ with $a b=0$ we have $\mathrm{c}_{a}\left(1-\mathrm{c}_{r} \mathrm{e}_{s}\right) f \mathrm{c}_{b}=0$, and since $R$ is $(S, \omega)$-Armendariz, it follows that

$$
\left[\mathrm{c}_{a}\left(1-\mathrm{c}_{r} \mathrm{e}_{s}\right)\right](s) \cdot \omega_{s}\left(\left(f \mathrm{c}_{b}\right)(1)\right)=0 .
$$

Hence $-a \cdot r \cdot \omega_{s}(b)=0$, and the $S$-compatibility of $R$ implies that $a r b=0$. 
Combining Proposition 4.3 and Example 2.2(iii) recovers [18, Lemma 2.3(2)].

In the proof of Theorem 4.12 we will need the following observation on semicommutative skew generalized power series rings.

LEMMA 4.4. Let $R$ be a ring, $(S, \leq)$ a strictly ordered monoid, and $\omega: S \rightarrow \operatorname{End}(R)$ a monoid homomorphism.

(i) If $R[[S, \omega]]$ is semicommutative, then $a b=0$ implies $a \omega_{s}(b)=0$ for all $a, b \in R$ and all $s \in S$.

(ii) If $R[[S, \omega]]$ is reversible, or if $R[[S, \omega]]$ is semicommutative and $S$ is a group, then $R$ is $S$-compatible.

Proof. Suppose that $R[[S, \omega]]$ is semicommutative. Given $a, b \in R$ and $s \in S$ such that $a b=0$, semicommutativity implies that $\mathrm{c}_{a} \mathrm{e}_{s} \mathbf{c}_{b}=0$, so $a \omega_{s}(b)=0$. This proves (i). If, in addition, $s$ has an inverse in $S$, then $a \omega_{s}(b)=0$ implies $a b=0$. Likewise, in the case where $R[[S, \omega]]$ is reversible,

$$
a \omega_{s}(b)=0 \Rightarrow \mathrm{c}_{a} \mathrm{e}_{s} \mathrm{c}_{b}=0 \Rightarrow \mathrm{c}_{b} \mathrm{c}_{a} \mathrm{e}_{s}=0 \Rightarrow b a=0 \Rightarrow a b=0 .
$$

This proves (ii).

Perhaps the greatest unsolved problem in noncommutative ring theory today is the Köthe conjecture, which posits that a ring with no nonzero nil ideals has no nonzero nil one-sided ideals. (See [36] for a discussion of the Köthe conjecture and various related problems.) The Köthe conjecture has been resolved in several special cases, including for rings with Krull dimension, for PI rings, and for algebras over uncountable fields. We will presently add $S$-compatible $(S, \omega)$-Armendariz rings to this list.

For a ring $R$, let $\mathfrak{N}(R)$ denote the set of nilpotent elements of $R, \mathrm{~N}_{0}(R)$ the Wedderburn radical of $R$ (that is, the sum of all nilpotent ideals of $R$ ), $\operatorname{Nil}_{*}(R)$ the prime radical of $R, \mathrm{Nil}^{*}(R)$ the upper nilradical of $R$, and $\mathrm{A}(R)$ the sum of all nil left ideals of $R$ (which coincides with the sum of all nil right ideals of $R$ ). The Köthe conjecture is equivalent to the statement that $A(R)$ is always nil, that is, $\mathrm{Nil}^{*}(R)=A(R)$ for every $\operatorname{ring} R$.

PROPOSITION 4.5. Let $R$ be a ring, $(S, \leq)$ a strictly ordered monoid, and $\omega: S \rightarrow$ $\operatorname{End}(R)$ a monoid homomorphism. If $R$ is $(S, \omega)$-Armendariz and $\omega_{s}$ is compatible for some $s \in S \backslash\{1\}$, then:

(i) for all $a, b \in R$ and $x \in \mathfrak{N}(R), a b=0$ implies ax $b=0$;

(ii) $\mathfrak{N}(R)$ is a (nonunital) subring of $R$;

(iii) $\mathrm{N}_{0}(R)=\mathrm{Nil}_{*}(R)=\mathrm{Nil}^{*}(R)=\mathrm{A}(R)$. In particular, the Köthe problem has $a$ positive solution in the class of $S$-compatible $(S, \omega)$-Armendariz rings.

Proof. (i) Since $\omega_{s}$ is compatible for some $s \in S \backslash\{1\}$, (i) follows directly from Lemma 4.2(iii).

(ii) Let $x, y \in \mathfrak{N}(R)$. Then $x^{n}=y^{n}=0$ for some $n \in \mathbb{N}$. Hence $(x y)^{n}=0$ by (i), and thus $x y \in \mathfrak{N}(R)$. Clearly $(x+y)^{2 n-1}$ is a finite sum of elements of the form $x^{\alpha_{1}} y^{\beta_{1}} x^{\alpha_{2}} y^{\beta_{2}} \cdots x^{\alpha_{k}} y^{\beta_{k}}$ for nonnegative integers $\alpha_{i}$ and $\beta_{i}$ satisfying $\sum_{i=1}^{k}\left(\alpha_{i}+\right.$ $\left.\beta_{i}\right)=2 n-1$. Then $\sum_{i=1}^{k} \alpha_{i} \geq n$ or $\sum_{i=1}^{k} \beta_{i} \geq n$, and thus $x^{\alpha_{1}+\alpha_{2}+\cdots+\alpha_{k}}=0$ 
or $y^{\beta_{1}+\beta_{2}+\cdots+\beta_{k}}=0$, and (i) implies that $x^{\alpha_{1}} y^{\beta_{1}} x^{\alpha_{2}} y^{\beta_{2}} \cdots x^{\alpha_{k}} y^{\beta_{k}}=0$. Hence $x+$ $y \in \mathfrak{N}(R)$, so $\mathfrak{N}(R)$ is a subring of $R$.

(iii) Let $x \in \mathrm{A}(R)$. Since $\mathrm{A}(R)$ is an ideal of $R$, and $\mathrm{A}(R) \subseteq \mathfrak{N}(R)$ by (ii), it follows that $R x R \subseteq \mathfrak{N}(R)$. Then $x^{n}=0$ for some $n \in \mathbb{N}$, and from (i) we deduce that $(R x R)^{2 n-1}=0$. Hence $x \in \mathrm{N}_{0}(R)$.

Combining Proposition 4.5(iii) and Example 2.2(i) recovers [18, Lemma 2.3(5)]. As a consequence of Propositions 4.3 and 4.5(iii) we obtain the following.

COROLlary 4.6. Let $R$ be a ring, $(S, \leq)$ a strictly ordered monoid, and $\omega: S \rightarrow$ $\operatorname{End}(R)$ a monoid homomorphism. If $R$ is $S$-compatible and $(S, \omega)$-Armendariz, and there exists $s \in S \backslash\{1\}$ such that $s^{m} \leq s^{n}$ for some positive integers $m<n$, then

$$
\mathrm{N}_{0}(R)=\mathrm{Nil}_{*}(R)=\mathrm{Nil}^{*}(R)=\mathrm{A}(R)=\mathfrak{N}(R) .
$$

Combining Corollary 4.6 and Example 2.2(iii) recovers [18, Lemma 2.3(6)].

A ring $R$ with the property that, for all $a_{0}, a_{1}, b_{0}, b_{1} \in R$, if $\left(a_{0}+a_{1} x\right)\left(b_{0}+\right.$ $\left.b_{1} x\right)=0$ in $R[x]$ then $a_{0} b_{1}=a_{1} b_{0}=0$ in $R$ has been called weak Armendariz by Lee and Wong in [22], and linearly Armendariz by Camillo and Nielsen in [7]. Camillo and Nielsen give a compelling argument in favour of the latter nomenclature in [7, p. 608], so we will follow their usage. Here we extend this condition to skew generalized power series rings.

Definition 4.7. Let $R$ be a ring, $(S, \leq)$ a strictly ordered monoid, and $\omega: S \rightarrow$ $\operatorname{End}(R)$ a monoid homomorphism. We say that $R$ is linearly $(S, \omega)$-Armendariz if for all $s \in S \backslash\{1\}$ and $a_{0}, a_{1}, b_{0}, b_{1} \in R$, whenever $\left(\mathrm{c}_{a_{0}}+\mathrm{c}_{a_{1}} \mathrm{e}_{s}\right)\left(\mathrm{c}_{b_{0}}+\mathrm{c}_{b_{1}} \mathrm{e}_{s}\right)=0$ in $R[[S, \omega]]$, then $a_{0} b_{0}=a_{0} b_{1}=a_{1} \cdot \omega_{s}\left(b_{0}\right)=a_{1} \cdot \omega_{s}\left(b_{1}\right)=0$ in $R$.

Obviously, all $(S, \omega)$-Armendariz rings are linearly $(S, \omega)$-Armendariz. However, as [22, Example 3.2] shows, a linearly $(S, \omega)$-Armendariz ring $R$ need not be $(S, \omega)$ Armendariz, even in the case where $R$ is commutative and $R[[S, \omega]]=R[x]$.

PROpOSITION 4.8. Let $R$ be a ring, $(S, \leq)$ a strictly ordered monoid, and $\omega: S \rightarrow$ $\operatorname{End}(R)$ a monoid homomorphism. The following conditions are equivalent:

(i) $\quad R$ is linearly $(S, \omega)$-Armendariz and reduced, and $\omega_{s}$ is injective for every $s \in S$;

(ii) $\quad R$ is $S$-rigid and $s^{2} \notin\{1, s\}$ for every $s \in S \backslash\{1\}$.

Proof. To prove that (i) implies (ii), we first show that $R$ is $S$-rigid. Let $a \in R$ and $s \in S$ be such that $a \omega_{s}(a)=0$. Since $R$ is reduced, $\omega_{s}(a) a=0$; thus,

$$
\left(\mathrm{c}_{\omega_{s}(a)}+\mathrm{C}_{-\omega_{s}(a)} \mathrm{e}_{s}\right)\left(\mathrm{c}_{a}+\mathrm{c}_{\omega_{s}(a)} \mathrm{e}_{s}\right)=0 .
$$

Since $R$ is linearly $(S, \omega)$-Armendariz, it follows that $\omega_{s}(a) \cdot \omega_{s}(a)=0$. Since $R$ is reduced, $\omega_{s}(a)=0$, and thus $a=0$ by the injectivity of $\omega_{s}$.

Now we show that for any $s \in S \backslash\{1\}, s^{2} \neq 1$ and $s^{2} \neq s$. If $s^{2}=1$, then $\left(\mathrm{C}_{1}+\right.$ $\left.\mathrm{C}_{-1} \mathrm{e}_{s}\right)\left(\mathrm{C}_{1}+\mathrm{C}_{1} \mathrm{e}_{s}\right)=0$ leads to $1 \cdot 1=0$, a contradiction. If $s^{2}=s$, then $\left(\mathrm{C}_{1}+\right.$ $\left.\mathrm{C}_{-1} \mathrm{e}_{s}\right) \mathrm{c}_{1} \mathrm{e}_{s}=0$, and again we obtain $1 \cdot 1=0$, a contradiction. 
We now prove that (ii) implies (i). Since $R$ is $S$-rigid, Lemma 2.5 implies that $R$ is reduced and $\omega_{s}$ is injective for every $s \in S$. To show that $R$ is linearly $(S, \omega)$ Armendariz, consider any $s \in S \backslash\{1\}$ and $a_{0}, a_{1}, b_{0}, b_{1} \in R$ with $\left(\mathrm{c}_{a_{0}}+\mathrm{c}_{a_{1}} \mathrm{e}_{s}\right)\left(\mathrm{c}_{b_{0}}+\right.$ $\left.\mathrm{C}_{b_{1}} \mathrm{e}_{s}\right)=0$. Since the elements $1, s$ and $s^{2}$ are different, it follows that

$$
a_{0} b_{0}=0, \quad a_{0} b_{1}+a_{1} \cdot \omega_{s}\left(b_{0}\right)=0, \quad a_{1} \cdot \omega_{s}\left(b_{1}\right)=0 .
$$

Since $R$ is reduced and $a_{0} b_{0}=0$, by multiplying the second equation of (4.1) by $b_{0}$ we obtain

$$
b_{0} a_{1} \cdot \omega_{s}\left(b_{0}\right)=0 \Rightarrow b_{0} a_{1} b_{0}=0 \Rightarrow a_{1} b_{0}=0 \Rightarrow a_{1} \cdot \omega_{s}\left(b_{0}\right)=0,
$$

using the compatibility of $\omega_{s}$. Therefore $R$ is linearly $(S, \omega)$-Armendariz.

We will say that an endomorphism $\sigma$ of a ring $R$ is idempotent-stabilizing if $\sigma(e)=e$ for every idempotent $e$ of $R$. In [13, Proposition 20] it was proved that for any $\sigma$-skew Armendariz ring $R$, the ring $R[[x ; \sigma]]$ is abelian if and only if $\sigma$ is idempotentstabilizing. The first part of the following result implies that the latter condition is always satisfied, and thus all $\sigma$-skew Armendariz rings are abelian. The second part of the result generalizes [17, Lemma 7], [15, Corollary 8], and [22, Lemma 3.4].

PROPOSITION 4.9. Let $R$ be a ring, $(S, \leq)$ a strictly ordered monoid, and $\omega: S \rightarrow$ $\operatorname{End}(R)$ a monoid homomorphism. If $R$ is linearly $(S, \omega)$-Armendariz, then:

(i) for every $s \in S$, the endomorphism $\omega_{s}$ is idempotent-stabilizing;

(ii) if $S$ is nontrivial, then $R$ is abelian.

Proof. (i) The statement is trivial if $s=1$, so assume that $s \in S \backslash\{1\}$. Notice that, as in the proof of [32, Lemma 4], for any idempotent $e \in R$,

$$
\left(\mathrm{c}_{1-e}+\mathrm{c}_{(1-e) \omega_{s}(e)} \mathrm{e}_{s}\right)\left(\mathrm{c}_{e}+\mathrm{c}_{(e-1) \omega_{s}(e)} \mathrm{e}_{s}\right)=0 .
$$

Since $R$ is linearly $(S, \omega)$-Armendariz, $0=(1-e) \omega_{s}(e)$. As the idempotent $e \in R$ was arbitrary, $0=e \omega_{s}(1-e)=e\left(1-\omega_{s}(e)\right)$. The equations $(1-e) \omega_{s}(e)=0$ and $e\left(1-\omega_{s}(e)\right)=0$ yield $\omega_{s}(e)=e$.

(ii) As suggested by the proof of [34, Lemma 2.4], let $a, e \in R$ with $e$ an idempotent and $s \in S \backslash\{1\}$ be given. Then

$$
\left(\mathrm{c}_{e}+\mathrm{c}_{e a(e-1)} \mathrm{e}_{s}\right)\left(\mathrm{c}_{1-e}+\mathrm{c}_{e a(1-e)} \mathrm{e}_{s}\right)=0,
$$

and since $R$ is linearly $(S, \omega)$-Armendariz, it follows that $e a(1-e)=0$. Hence $e R(1-e)=\{0\}$ for any idempotent $e \in R$, which proves that $R$ is abelian.

This justifies the placement of ' $(S, \omega)$-Armendariz' in the chart at the beginning of Section 4. Note that the $(S, \omega)$-Armendariz condition does not imply any of the conditions in the first two rows of that chart, in general. For instance, [7, Example 9.3] shows that a ring can be $(S, \omega)$-Armendariz but not 2-primal.

The following proposition shows that for any $(S, \omega)$-Armendariz ring $R$ the set of all idempotents of $R[[S, \omega]]$ coincides with that of $R$. The proposition generalizes [13, Lemma 19]. 
PRoposition 4.10. Let $R$ be a ring, $(S, \leq)$ a strictly ordered monoid, and $\omega: S \rightarrow$ $\operatorname{End}(R)$ a monoid homomorphism. Assume that $R$ is $(S, \omega)$-Armendariz.

(i) If $f$ is an idempotent of $R[[S, \omega]]$, then $f(1)$ is an idempotent of $R$ and $f=c_{f(1)}$.

(ii) $R[[S, \omega]]$ is abelian.

Proof. (i) Since $f(f-1)=0$ and $R$ is $(S, \omega)$-Armendariz, we obtain $f(1)$. $(f(1)-1)=0$, and so $f(1)$ is an idempotent of $R$. Moreover, for any $s \in S \backslash\{1\}$ we have $0=f(1) \cdot(f-1)(s)=f(1) f(s)$. On the other hand, since $(f-1) f=0$, it follows that $(f(1)-1) \cdot f(s)=0$, and thus $f(s)=f(1) f(s)=0$. Hence $f=\mathrm{c}_{f(1)}$.

(ii) Let $f=f^{2} \in R[[S, \omega]]$. Then by (i), $f=\mathrm{c}_{e}$ for some idempotent $e$ of $R$. Hence by Proposition 4.9(i), $\omega_{s}(e)=e$ for every $s \in S$. Furthermore, by Proposition 4.9(ii), $e$ is central in $R$. Now it is easy to see that $\mathrm{c}_{e} g=g \mathrm{C}_{e}$ for every $g \in R[[S, \omega]]$.

We now turn to reduced $(S, \omega)$-Armendariz rings. We will characterize such rings in Theorem 4.12 below in the case where $S$ belongs to a subclass of the class of unique product monoids.

Recall that a monoid $S$ is called a unique product monoid (or a u.p. monoid, or u.p.) if for any two nonempty finite subsets $X, Y \subseteq S$ there exist $x \in X$ and $y \in Y$ such that $x y \neq x^{\prime} y^{\prime}$ for every $\left(x^{\prime}, y^{\prime}\right) \in X \times Y \backslash\{(x, y)\}$; the element $x y$ is called a u.p. element of $X Y=\{s t: s \in X, t \in Y\}$. Unique product monoids and groups play an important role in ring theory, for example providing a positive case in the zero-divisor problem for group rings (see also [5]), and their structural properties have been extensively studied (see [9] and references therein, or [35]). The class of u.p. monoids includes the right and the left totally ordered monoids, submonoids of a free group, and torsionfree nilpotent groups.

For our purposes, the following, more stringent conditions are needed.

Definition 4.11. Let $(S, \leq)$ be an ordered monoid. We say that $(S, \leq)$ is an artinian narrow unique product monoid (or an a.n.u.p. monoid, or simply a.n.u.p.) if for every two artinian and narrow subsets $X$ and $Y$ of $S$ there exists a u.p. element in the product $X Y$. We say that $(S, \leq)$ is quasitotally ordered (and that $\leq$ is a quasitotal order on $S$ ) if $\leq$ can be refined to an order $\preccurlyeq$ with respect to which $S$ is a strictly totally ordered monoid.

For any ordered monoid $(S, \leq)$, the following chain of implications holds:

$S$ is commutative, torsion-free, and cancellative

$\Downarrow$

$(S, \leq)$ is quasitotally ordered

$\Downarrow$

$(S, \leq)$ is a.n.u.p.

$\Downarrow$

$S$ is u.p. 
The converse of the bottom implication holds if $\leq$ is the trivial order. For more details, examples, and interrelationships between these and other conditions on ordered monoids, we refer the reader to [29].

The following theorem generalizes [8, Theorem 1 and Corollaries 2 and 3], [13, Proposition 3 and Corollary 4], [20, Corollary 3.5], [24, Lemmas 2.1 and 3.1], [25, Corollary 1.2], [30, Theorem A] and [32, Theorem 6].

THEOREM 4.12. Let $R$ be a ring, $(S, \leq)$ a strictly ordered monoid, and $\omega: S \rightarrow$ $\operatorname{End}(R)$ a monoid homomorphism. Consider the following six conditions:

(i) $\quad R$ is reduced, and whenever $f, g \in R[[S, \omega]]$ satisfy $f g=0$, then $f(s) g(t)=0$ for all $s, t \in S$;

(ii) $R$ is $(S, \omega)$-Armendariz and reduced, and $\omega_{s}$ is injective for every $s \in S$;

(iii) $R$ is linearly $(S, \omega)$-Armendariz and reduced, and $\omega_{s}$ is injective for every $s \in S$;

(iv) $R[[S, \omega]]$ is reduced;

(v) $R$ is semiprime, and the ring $R[[S, \omega]]$ is reversible;

(vi) $R$ is $S$-rigid.

Then:

$$
\begin{array}{ccccc}
\text { (i) } & \Leftrightarrow & \text { (ii) } & \Rightarrow & \text { (iii) } \\
\Downarrow & & & & \Downarrow \\
\text { (iv) } & \Rightarrow & \text { (v) } & \Rightarrow & \text { (vi) }
\end{array}
$$

If $(S, \leq)$ is a.n.u.p., then all six conditions are equivalent.

PROOF. First assume that $(S, \leq)$ is strictly ordered but not necessarily a.n.u.p.

(ii) $\Rightarrow$ (i). Assume (ii). Let $f, g \in R[[S, \omega]]$ be such that $f g=0$, and let $s, t \in S$. We must show that $f(s) g(t)=0$. Since $R$ is $(S, \omega)$-Armendariz, $f(s) \cdot \omega_{s}(g(t))=0$, so the case $s=1$ is done. Suppose $s \neq 1$, and set $r=f(s) g(t)$. Because $R$ is semicommutative, $r \omega_{s}(r)=0$; because $R$ is reversible, $\omega_{s}(r) r=0$. Hence for $h=$ $\mathrm{C}_{\omega_{s}(r)}+\mathrm{c}_{\omega_{s}(r)} \mathrm{e}_{s}$ and $k=\mathrm{c}_{r}-\mathrm{c}_{\omega_{s}(r)} \mathrm{e}_{s}$ in $R[[S, \omega]]$ we have $h k=0$. The $(S, \omega)$ Armendariz hypothesis implies that $0=h(s) \cdot \omega_{s}(k(1))=\omega_{s}(r)^{2}$; now, since $R$ is reduced and $\omega_{s}$ is injective, $0=r=f(s) g(t)$.

(ii) $\Rightarrow$ (iii). Trivial.

(iii) $\Rightarrow$ (vi). Proposition 4.8 .

(i) $\Rightarrow$ (iv). For any $f \in R[[S, \omega]]$, if $f^{2}=0$ then (i) implies that $f=0$.

(iv) $\Rightarrow$ (v). Trivial.

(v) $\Rightarrow$ (vi). Since $R$ is reversible and semiprime, it is reduced. Since $R$ is $S$-compatible by Lemma 4.4(ii), it is $S$-rigid by Lemma 2.5(iii).

(i) $\Rightarrow$ (ii). It is easy to see that if $R$ is $S$-compatible and (i) holds, then (ii) holds. We have already shown that (i) implies (vi). From (vi) and Lemma 2.5(iii), we infer that $R$ is $S$-compatible.

Now assume that $(S, \leq)$ is a.n.u.p. That (vi) implies (ii) follows from Lemma 2.5(iii) and [29, Proposition 3.6]; however, in the interests of a self-contained presentation, we will give an alternative, direct proof. Suppose that there exist $f, g \in R[[S, \omega]]$ such that $f g=0$ and $f(s) \cdot \omega_{s}(g(t)) \neq 0$ for some $s, t \in S$. Since 
by Lemma 2.5(iii) the ring $R$ is reduced, the intersection of all minimal prime ideals of $R$ is equal to (0). Hence there exists a minimal prime ideal $P$ of $R$ such that $f(s) \cdot \omega_{s}(g(t)) \notin P$, and thus the sets

$$
X=\{x \in S: f(x) \notin P\} \quad \text { and } \quad Y=\left\{y \in S:(\exists x \in S) \omega_{x}(g(y)) \notin P\right\}
$$

are nonempty. Since $X \subseteq \operatorname{supp}(f)$ and $Y \subseteq \operatorname{supp}(g), X$ and $Y$ are artinian and narrow subsets of $S$, and since $S$ is an a.n.u.p. monoid, there exists $(a, b) \in X \times Y$ such that $a b$ is a u.p. element of $X Y$. Since $f g=0$,

$$
0=(f g)(a b)=f(a) \cdot \omega_{a}(g(b))+\sum_{(u, v) \in \mathrm{X}_{a b}(f, g) \backslash\{(a, b)\}} f(u) \cdot \omega_{u}(g(v)) .
$$

Observe that if $(u, v) \in \mathrm{X}_{a b}(f, g) \backslash\{(a, b)\}$, then since $a b$ is a u.p. element of $X Y$, we have $u \notin X$ or $v \notin Y$, and thus $f(u) \cdot \omega_{u}(g(v)) \in P$. Hence (4.2) implies that $f(a) \cdot \omega_{a}(g(b)) \in P$. Since each minimal prime ideal of a reduced ring is completely prime (see [21, Lemma 12.6]) and $a \in X$, it follows that $\omega_{a}(g(b)) \in P$. It is not hard to show that, since $R$ is reduced, for every $r \in R$ and every $n \in \mathbb{N}$ we have $\operatorname{ann}_{r}^{R}(r)=\operatorname{ann}_{\ell}^{R}(r)=\operatorname{ann}_{r}^{R}\left(r^{n}\right)=\operatorname{ann}_{\ell}^{R}\left(r^{n}\right)$. Therefore, if $\operatorname{ann}_{\ell}^{R}\left(\omega_{a}(g(b))\right) \subseteq P$, then the set

$$
Z=\left\{z_{1} z_{2} \cdots z_{m}: m \in \mathbb{N}, z_{i}=\omega_{a}(g(b)) \text { or } z_{i} \in R \backslash P \text { for each } i\right\}
$$

would be a multiplicatively closed set disjoint from $\{0\}$ and properly containing $R \backslash P$, which contradicts $P$ being a minimal prime. Therefore $\operatorname{ann}_{\ell}^{R}\left(\omega_{a}(g(b))\right) \nsubseteq P$, so $t \cdot \omega_{a}(g(b))=0$ for some $t \in R \backslash P$. Because $R$ is $S$-compatible, we have $t$. $\omega_{x}(g(b))=0$ for every $x \in S$, whence $b \notin Y$. This final contradiction proves that $R$ is $(S, \omega)$-Armendariz, establishing (ii).

EXAMPLE 4.13. The following counterexamples delimit Theorem 4.12. In particular, these show that when $(S, \leq)$ is not a.n.u.p., the only implications between the six listed conditions are the ones stated in the theorem or following from the stated implications by transitivity.

(i) Let $R$ be a field of characteristic char $R \neq 2$, let $S=\{1, s\}$ be the two-element group (with the trivial order), and let $\omega: S \rightarrow \operatorname{End}(R)$ be the trivial map. Clearly $R[[S, \omega]]$ is reduced; however, the equation $\left(1-\mathrm{e}_{s}\right)\left(1+\mathrm{e}_{s}\right)=0$ shows that $R$ is not linearly $(S, \omega)$-Armendariz. Thus, (iv) $\not \Rightarrow$ (iii) in Theorem 4.12 in general.

(ii) Let $R[[S, 1]]$ be the (skew) generalized power series ring constructed in [29, Example 3.3]; namely, $R$ is a field of characteristic 2, and $(S, \leq)$ is the u.p. but not a.n.u.p. monoid constructed in [29, Example 2.6]. In [29, Example 3.3] two elements $f, g \in R[[S, 1]]$ were constructed with the property that $f g=0$. One can easily verify that $g f \neq 0$; therefore, $R[[S, 1]]$ is not reversible. Since $S$ is a u.p. monoid and $R$ is $S$-rigid, Proposition 4.8 shows that $R$ is linearly $(S, 1)$ Armendariz. Thus, (iii) $\nRightarrow(\mathrm{v})$ in Theorem 4.12 in general. 
(iii) Let $R=\mathbb{F}_{2}$, let $S=Q_{8}$, the quaternion group of order 8 , with the trivial order, and let $\omega: S \rightarrow \operatorname{End}(R)$ be the trivial map. Then $R[[S, \omega]]$ is the group algebra $R S$, which by [27, Example 7] is reversible but not reduced. Thus, (v) $\not$ (iv) in Theorem 4.12 in general.

(iv) Let $R=\mathbb{F}_{2}$, let $S=D_{8}$, the dihedral group of order 8, with the trivial order, and let $\omega: S \rightarrow \operatorname{End}(R)$ be the trivial map. Then $R[[S, \omega]]$ is the group algebra $R S$, which by [27, p. 316] is not reversible. Letting $s \in S$ be any element of order 2 , then $\left(1+\mathrm{e}_{s}\right)\left(1+\mathrm{e}_{s}\right)=0$; therefore, $R$ is not linearly $(S, \omega)$-Armendariz. Obviously $R$ is $S$-rigid. Thus, (vi) $\not \Rightarrow$ (iii) and (vi) $\not \Rightarrow(v)$ in Theorem 4.12 in general.

(v) Let $R$ be a uniserial artinian ring that is not a division ring possessing a nonidentity automorphism $\sigma \in \operatorname{Aut}(R)$. (For an explicit example, one could let $R=F[x] /(p(x))^{k}$ where $F$ is a field, $p(x) \in F[x]$ is an irreducible polynomial with $\operatorname{deg}(p(x))>1$, and $k \geq 2$. Putting $E=F[x] /(p(x))$, the ring $R$ becomes a $k$-dimensional $E$-algebra, and any nontrivial element of the Galois group $\operatorname{Gal}(E / F)$ naturally induces a nontrivial automorphism of $R$.) Let $S=\mathbb{N} \cup\{0\}$ under addition, with the trivial order, and define $\omega: S \rightarrow \operatorname{End}(R)$ by $\omega(n)=$ $\sigma^{n}$. Note that $S$ is quasitotally ordered. By [28, Proposition 3.5], the ring $R[[S, \omega]] \cong R[x ; \sigma]$ is reversible, though $R$ and $R[[S, \omega]]$ are not reduced. This shows that in Theorem 4.12 the hypothesis that $R$ be reduced cannot be dropped from condition (i) or (ii), and the hypothesis that $R$ be semiprime cannot be dropped from condition (v).

It is of interest to consider a condition absent from Theorem 4.12: semicommutativity. Semicommutativity is of course a central issue for Armendariz rings (and generalizations thereof), dating back to their inception: see [37, Section 4]. Theorem 4.12 tells us that under appropriate circumstances the reduced condition on a skew generalized power series ring is equivalent to the generally weaker reversible condition. What about the still weaker semicommutative condition? Theorem 4.1 apparently has no bearing on this problem, since the hypotheses of Theorem 4.1 already entail most of condition (ii) of Theorem 4.12. This leaves us with the following open question.

Question 4.14. Suppose $R$ is a semiprime $\operatorname{ring},(S, \leq)$ is a strictly ordered a.n.u.p. monoid, and $\omega: S \rightarrow \operatorname{End}(R)$ is a monoid homomorphism. If the skew generalized power series ring $R[[S, \omega]]$ is semicommutative, must $R[[S, \omega]]$ be reversible (and therefore reduced)?

A power series ring over a 2-primal ring need not be 2-primal, as examples in $[14,26]$ show. Nevertheless, under appropriate conditions, a skew generalized power series ring will be 2-primal.

THEOREM 4.15. Let $R$ be a ring, $(S, \leq)$ a strictly ordered a.n.u.p. monoid, and $\omega: S \rightarrow \operatorname{End}(R)$ a monoid homomorphism. Suppose that $\mathrm{Nil}_{*}(R)$ is a nilpotent ideal, and suppose that for every $s \in S$ and every $a \in R$, if $a R \omega_{S}(a) \subseteq \mathrm{Nil}_{*}(R)$, then $a$ is 
nilpotent. Then the skew generalized power series ring $R[[S, \omega]]$ is 2-primal if and only if $R$ is 2-primal.

PRoOF. If the ring $A=R[[S, \omega]]$ is 2-primal, then $R$ is 2-primal by [4, Proposition 2.2].

Conversely, suppose that $R$ is 2-primal, and assume the prime radical $I=\operatorname{Nil}_{*}(R)$ satisfies $I^{n}=(0)$ for some $n \in \mathbb{N}$. Let $\pi: R \rightarrow R / I=\bar{R}$ be the canonical map. Since $R$ is 2-primal, its prime radical is $S$-invariant. Therefore $J=I[[S, \omega]]$ is an ideal of $A$, and we have a monoid homomorphism $v: \operatorname{End}(R) \rightarrow \operatorname{End}(\bar{R})$ given by $v(\tau)(\bar{x})=$ $\overline{\tau(x)}$ for each $\bar{x} \in \bar{R}$. The surjective ring homomorphism $A \rightarrow \bar{R}[[S, v \circ \omega]]$ given by $f \mapsto \pi \circ f$ induces an isomorphism $A / J \cong \bar{R}[[S, v \circ \omega]]$.

Suppose that for $s \in S$ and $a \in R$ we have $\bar{a} \cdot(v \circ \omega)_{s}(\bar{a})=\overline{0}$ in $\bar{R}$. Since $R$ is 2-primal, $\bar{R}$ is reduced and hence semicommutative; therefore, $\bar{a} \cdot \bar{R} \cdot(v \circ \omega)_{s}(\bar{a})=$ $\{\overline{0}\}$, whence $a R \omega_{s}(a) \subseteq I$. By hypothesis, then, $a$ is nilpotent, so $\bar{a}=\overline{0}$ in $\bar{R}$. We have shown that $(v \circ \omega)_{s}$ is a rigid endomorphism of $\bar{R}$, for arbitrary $s \in S$.

Thus, by Theorem 4.12, the $\operatorname{ring} A / J \cong \bar{R}[[S, v \circ \omega]]$ is reduced. Clearly $J^{n}=(0)$, so $A$ is 2-primal.

REMARK 4.16. In Theorem 4.15, if $\omega$ is trivial then the condition ' $a R \omega_{s}(a) \subseteq$ $\mathrm{Nil}_{*}(R)$ implies $a$ nilpotent' is vacuous. So [24, Theorem 2.3] is a special case of Theorem 4.15 .

Let $R$ be a semiprime left Goldie ring, and let $\mathcal{C}$ denote the set of regular elements of $R$ (that is, elements that are neither left nor right zero-divisors). If $\sigma \in \operatorname{End}(R)$ is injective, then $\sigma(\mathcal{C}) \subseteq \mathcal{C}$ by [16, Proposition 2.4]. Therefore, if $Q=Q_{\mathrm{cl}}^{\ell}(R)$ is the classical left ring of quotients of $R$, then one can verify that $\sigma$ extends (uniquely) to an endomorphism $\tilde{\sigma}$ of $Q$ defined by $\widetilde{\sigma}\left(b^{-1} a\right)=\sigma(b)^{-1} \sigma(a)$ for all $a \in R$ and $b \in \mathcal{C}$.

In this setting, if $S$ is a monoid and $\omega: S \rightarrow \operatorname{End}(R)$ is a monoid homomorphism such that $\omega_{s}$ is injective for every $s \in S$, then there is an induced monoid homomorphism $\widetilde{\omega}: S \rightarrow \operatorname{End}(Q)$ defined by

$$
\widetilde{\omega}_{s}=\widetilde{\omega}_{s} \text { for each } s \in S .
$$

Notice that $\widetilde{\omega}_{s}$ is injective for every $s \in S$.

The following result generalizes [32, Theorem 10].

THEOREM 4.17. Let $R$ be a semiprime left Goldie ring, $(S, \leq)$ a nontrivial strictly ordered a.n.u.p. monoid, and $\omega: S \rightarrow \operatorname{End}(R)$ a monoid homomorphism such that $\omega_{s}$ is injective for every $s \in S$. Let $Q=Q_{\mathrm{cl}}^{\ell}(R)$ denote the classical left ring of quotients of $R$, and $\widetilde{\omega}: S \rightarrow \operatorname{End}(Q)$ the induced $S$-action. Then the following conditions are equivalent:

(i) $\quad R$ is $(S, \omega)$-Armendariz;

(ii) $R$ is linearly $(S, \omega)$-Armendariz;

(iii) $R$ is $(S, \omega)$-rigid;

(iv) $Q$ is $(S, \widetilde{\omega})$-Armendariz; 
(v) $Q$ is linearly $(S, \widetilde{\omega})$-Armendariz;

(vi) $Q$ is $(S, \widetilde{\omega})$-rigid.

PROOF. (i) $\Rightarrow$ (ii). Trivial.

(ii) $\Rightarrow$ (v). We have to show that for any $p_{0}, p_{1}, q_{0}, q_{1} \in Q$ and $s \in S \backslash\{1\}$,

if $\left(\mathrm{c}_{p_{0}}+\mathrm{c}_{p_{1}} \mathrm{e}_{s}\right)\left(\mathrm{c}_{q_{0}}+\mathrm{c}_{q_{1}} \mathrm{e}_{s}\right)=0$ in $Q[[S, \widetilde{\omega}]]$, then $p_{0} q_{1}=p_{1} \cdot \widetilde{\omega}_{s}\left(q_{0}\right)=0$.

Now, there exist $a_{0}, a_{1}, b_{0}, b_{1}, u \in R$ such that $u$ is regular and $p_{i}=u^{-1} a_{i}, q_{i}=$ $u^{-1} b_{i}$ for $i=1,2$. Furthermore, for some $d_{0}, d_{1}, v \in R$ with $v$ regular, we can write $a_{0} u^{-1}=v^{-1} d_{0}$ and $a_{1} \omega_{s}(u)^{-1}=v^{-1} d_{1}$. Now it is easy to see that in $R[[S, \omega]]$ we have $\left(\mathrm{c}_{d_{0}}+\mathrm{c}_{d_{1}} \mathrm{e}_{s}\right)\left(\mathrm{c}_{b_{0}}+\mathrm{c}_{b_{1}} \mathrm{e}_{s}\right)=0$. Since $R$ is linearly $(S, \omega)$ Armendariz, we obtain $d_{0} b_{1}=d_{1} \cdot \omega_{s}\left(b_{0}\right)=0$. Now $p_{0} q_{1}=p_{1} \cdot \widetilde{\omega}_{s}\left(q_{0}\right)=0$ follows easily, proving (4.3).

(iv) $\Leftrightarrow$ (v). Trivial.

(v) $\Leftrightarrow$ (vi). Assuming (v), Proposition 4.9(ii) implies that $Q$ is abelian; being semisimple, $Q$ is reduced. Hence (vi) holds by Theorem 4.12.

(vi) $\Rightarrow$ (iii). Trivial.

(iii) $\Rightarrow$ (i). This follows from Theorem 4.12.

Applying Theorem 4.17 to Example 2.2(i), we obtain the following improvement of [17, Proposition 18] (which was, in turn, an improvement of [1, Theorem 7]), recovering [22, Theorem 3.3].

Corollary 4.18. Suppose that $R$ is a semiprime left Goldie ring. Then $R$ is Armendariz if and only if $R$ is reduced.

To illustrate this corollary, we observe that (perhaps inevitably) throughout the literature most proofs that particular rings are Armendariz run afoul of Poincaré's counsel: 'Il faut triompher par la pensée et non par le calcul'. Let $F=k\langle x, y\rangle$ be the free algebra on two noncommuting indeterminates over a field $k$, and consider the two factor rings $R_{1}=F / F x^{2} F$ and $R_{2}=F /\left(F x^{2} F+F y^{2} F\right)$. Both $R_{1}$ and $R_{2}$ are prime rings, and one can directly check that $R_{1}$ is Armendariz but $R_{2}$ is not. In fact, $R_{1}$ is a construction of Camillo and Nielsen in [7, Example 9.3], apparently the first example in the literature of an Armendariz ring that is not 2-primal. Camillo and Nielsen's proof that $R_{1}$ is Armendariz is based on an intricate calculation of zero-divisors. In contrast, Corollary 4.18 provides a 'structural' proof that $R_{2}$ is not Armendariz: it is noetherian and prime but not reduced.

\section{The $(S, \omega)$-Armendariz condition and ring extensions}

It is easy to see that if $I$ is a reduced ideal of a ring $R$ (that is, $I$ is an ideal of $R$ such that $x^{2}=0$ implies that $x=0$ for every $x \in I$ ), then for any $a, b \in R, a b=0$ implies that $a I b=\{0\}$. We will use this observation freely in the following proof. 
PROPOSITION 5.1. Let $R$ be a ring, $(S, \leq)$ a strictly totally ordered monoid and $\omega: S \rightarrow \operatorname{End}(R)$ a monoid homomorphism such that $R$ is $S$-compatible. Let $f, g \in$ $R[[S, \omega]]$ be such that for some reduced ideal I of $R$,

$$
(f g)(x y)=0 \Rightarrow f(x) \cdot \omega_{x}(g(y)) \in I \quad \text { for any } x, y \in S .
$$

Then for any $s \in S$ the following are equivalent:

(i) $f(x) \cdot \omega_{x}(g(y))=0$ for any $x, y \in S$ such that $x y \leq s$;

(ii) $(f g)(z)=0$ for any $z \leq s$.

PROOF. That (i) implies (ii) is obvious (and requires no assumptions about the order $\leq$ or the existence of a reduced ideal $I$ ).

To prove that (ii) implies (i), let us suppose that the implication fails. Since the sets $\operatorname{supp}(f)$ and $\operatorname{supp}(g)$ are well-ordered, we can choose an element $\left(x_{0}, y_{0}\right) \in S \times S$ minimal with respect to the lexicographic order such that

$$
x_{0} y_{0} \leq s \quad \text { and } \quad f\left(x_{0}\right) \cdot \omega_{x_{0}}\left(g\left(y_{0}\right)\right) \neq 0 .
$$

Hence there exist $n \in \mathbb{N}$ and $\left(x_{1}, y_{1}\right), \ldots,\left(x_{n}, y_{n}\right) \in S \times S \backslash\left\{\left(x_{0}, y_{0}\right)\right\}$ such that

$$
0=(f g)\left(x_{0} y_{0}\right)=\sum_{i=0}^{n} f\left(x_{i}\right) \cdot \omega_{x_{i}}\left(g\left(y_{i}\right)\right)
$$

and for each $i \in\{0,1, \ldots, n\}$ we have $x_{i} y_{i}=x_{0} y_{0}$ and $f\left(x_{i}\right) \cdot \omega_{x_{i}}\left(g\left(y_{i}\right)\right) \neq 0$. By the choice of $\left(x_{0}, y_{0}\right)$, for each $i \geq 1$ we have $x_{0}<x_{i}$, hence $y_{i}<y_{0}$, and thus $f\left(x_{0}\right) \cdot \omega_{x_{0}}\left(g\left(y_{i}\right)\right)=0$. Now the compatibility of $\omega_{x_{0}}$ and $\omega_{x_{i}}$ implies that $f\left(x_{0}\right)$. $\omega_{x_{i}}\left(g\left(y_{i}\right)\right)=0$. Hence for every $i \geq 1$ we have $f\left(x_{0}\right) \cdot I \cdot \omega_{x_{i}}\left(g\left(y_{i}\right)\right)=0$, and since $f\left(x_{0}\right) \cdot \omega_{x_{0}}\left(g\left(y_{0}\right)\right) \in I$ by assumption, multiplying Equation (5.1) on the left by $\left[f\left(x_{0}\right) \cdot \omega_{x_{0}}\left(g\left(y_{0}\right)\right)\right]^{2}$ yields $0=\left[f\left(x_{0}\right) \cdot \omega_{x_{0}}\left(g\left(y_{0}\right)\right)\right]^{3}$. Since $I$ is reduced, it follows that $f\left(x_{0}\right) \cdot \omega_{x_{0}}\left(g\left(y_{0}\right)\right)=0$, a contradiction.

As a corollary of the above result we obtain the following generalization of [25, Proposition 1.4].

COROllary 5.2. Let $R$ be a ring, $(S, \leq)$ a quasitotally ordered monoid and $\omega$ : $S \rightarrow \operatorname{End}(R)$ a monoid homomorphism. Assume that $R$ is $S$-compatible, and that there exists a reduced ideal I of $R$ such that for any $f, g \in R[[S, \omega]]$, if $f g=0$, then $f(s) \cdot \omega_{s}(g(t)) \in I$ for all $s, t \in S$. Then $R$ is $(S, \omega)$-Armendariz.

PROOF. By hypothesis, the order $\leq$ can be refined to a strict total order $\preccurlyeq$. Since Proposition 5.1 implies that $R$ is $(S, \omega, \preccurlyeq)$-Armendariz, Lemma 2.7 completes the proof.

As an immediate consequence of Corollary 5.2, we obtain the following extension property for $(S, \omega)$-Armendariz rings.

COROLlARY 5.3. Let $R$ be a ring, $(S, \leq)$ a quasitotally ordered monoid and $\omega: S \rightarrow$ $\operatorname{End}(R)$ a monoid homomorphism. Suppose that $R$ is $S$-compatible and that there 
exists an $S$-invariant, reduced ideal $I \subseteq R$ such that the factor ring $R / I$ is $(S, \bar{\omega})$ Armendariz, where $\bar{\omega}: S \rightarrow \operatorname{End}(R / I)$ is the induced monoid homomorphism. Then $R$ is $(S, \omega)$-Armendariz.

In [1, Theorem 5] Anderson and Camillo prove that for any ring $R$ and any integer $n \geq 2$, the factor ring $R[x] /\left(x^{n}\right)$ is Armendariz if and only if $R$ is reduced. As we will see in Corollary 5.6, Anderson and Camillo's result is a consequence of the following theorem.

THEOREM 5.4. Let $R$ be a ring, $(S, \leq)$ a strictly well-ordered monoid and $\omega: S \rightarrow$ $\operatorname{End}(R)$ a monoid homomorphism. Fix $s \in S$. Then the set

$$
I_{s}=\{f \in R[[S, \omega]]: f(x)=0 \text { for every } x \leq s\}
$$

is a proper ideal of $R[[S, \omega]]$. Assume that $\left(T, \leq^{\prime}\right)$ is a nontrivial quasitotally ordered monoid, and consider the following conditions:

(i) $\omega_{x}$ is rigid for every $x \in S$ satisfying $x \leq s$;

(ii) $R[[S, \omega]] / I_{S}$ is $\left(T, 1, \leq^{\prime}\right)$-Armendariz;

(iii) $R[[S, \omega]] / I_{S}$ is $\left(T, 1, \leq^{\prime \prime}\right)$-Armendariz where $\leq^{\prime \prime}$ is the trivial order;

(iv) $R[[S, \omega]] / I_{S}$ is linearly $\left(T, 1, \leq^{\prime \prime}\right)$-Armendariz where $\leq^{\prime \prime}$ is the trivial order.

In general,

$$
\text { (i) } \Rightarrow \text { (ii) } \Rightarrow \text { (iii) } \Rightarrow \text { (iv). }
$$

Now assume, moreover, that $s \neq 1$ and either of the following conditions holds:

(a) for every $x \in S \backslash\{1\}$ such that $x \leq s, \omega_{x}$ is injective and there exists $n \in \mathbb{N}$ such that $s<x^{n}$; or

(b) $\omega$ is trivial.

Then

$$
\text { (i) } \Leftrightarrow \text { (ii) } \Leftrightarrow \text { (iii) } \Leftrightarrow \text { (iv). }
$$

Proof. Since $(S, \leq)$ is a well-ordered monoid, $1 \leq x$ for all $x \in S$, and it easily follows that $I_{s}$ is a proper ideal of $R[[S, \omega]]$.

(i) $\Rightarrow$ (ii). By Lemma 2.7, without loss of generality we can assume that $\leq^{\prime}$ is already a total order. Suppose that $R[[S, \omega]] / I_{S}$ is not $\left(T, 1, \leq^{\prime}\right)$-Armendariz. Pick $F, G \in\left(R[[S, \omega]] / I_{S}\right)[[T, 1]]$ such that $F G=0$ and $F(t) G\left(t^{\prime}\right) \neq 0$ for some $t, t^{\prime} \in T$. For any $z \in T$ we have $F(z), G(z) \in R[[S, \omega]] / I_{S}$ and thus there exist $f_{z}, g_{z} \in R[[S, \omega]]$ such that $F(z)=\overline{f_{z}}$ and $G(z)=\overline{g_{z}}$ (where a bar denotes images modulo $I_{S}$ ). We will retain this notation for the remainder of the proof. Whenever $F(z)$ (respectively $G(z)$ ) is 0 , we will choose $f_{z}=0$ (respectively $g_{z}=0$ ).

Define the following total order $\preccurlyeq$ on $S \times S \times T \times T$ :

$$
\left(s_{1}, s_{2}, t_{1}, t_{2}\right) \prec\left(s_{3}, s_{4}, t_{3}, t_{4}\right) \Leftrightarrow\left\{\begin{array}{l}
s_{1} s_{2}<s_{3} s_{4}, \text { or } \\
s_{1} s_{2}=s_{3} s_{4} \text { and } s_{1}<s_{3}, \text { or } \\
s_{1}=s_{3} \text { and } s_{2}=s_{4} \text { and } t_{1}<^{\prime} t_{3}, \text { or } \\
s_{1}=s_{3} \text { and } s_{2}=s_{4} \text { and } t_{1}=t_{3} \text { and } t_{2}<^{\prime} t_{4} .
\end{array}\right.
$$


Then $S \times S \times \operatorname{supp}(F) \times \operatorname{supp}(G)$ is well-ordered under $\preccurlyeq$, so there exists $(x, y, c, d) \in S \times S \times \operatorname{supp}(F) \times \operatorname{supp}(G)$ minimal for the property $f_{c}(x) \cdot \omega_{x}\left(g_{d}\right.$ $(y)) \neq 0$. Since $f_{t} g_{t^{\prime}} \notin I_{s}$, we have $x y \leq s$.

We claim that for any element $\left(x^{\prime}, y^{\prime}, c^{\prime}, d^{\prime}\right) \in S \times S \times T \times T$,

$$
\begin{aligned}
x^{\prime} y^{\prime} & =x y \quad \text { and } \quad c^{\prime} d^{\prime}=c d \\
& \Rightarrow f_{c^{\prime}}\left(x^{\prime}\right) \cdot \omega_{x^{\prime}}\left(g_{d^{\prime}}\left(y^{\prime}\right)\right) \cdot f_{c}(x)=0 \quad \text { or }(x, y, c, d)=\left(x^{\prime}, y^{\prime}, c^{\prime}, d^{\prime}\right)
\end{aligned}
$$

Indeed, it suffices to consider $\left(x^{\prime}, y^{\prime}, c^{\prime}, d^{\prime}\right) \in S \times S \times \operatorname{supp}(F) \times \operatorname{supp}(G)$ for which $(x, y, c, d) \prec\left(x^{\prime}, y^{\prime}, c^{\prime}, d^{\prime}\right)$. Then either $x<x^{\prime}$, or else $x=x^{\prime}$ and $y=y^{\prime}$ and $c<^{\prime} c^{\prime}$. If $x<x^{\prime}$, then $x y^{\prime}<x y$, hence $\left(x, y^{\prime}, c, d^{\prime}\right) \prec(x, y, c, d)$. If $x=x^{\prime}$ and $y=y^{\prime}$ and $c<^{\prime} c^{\prime}$, then $c d=c^{\prime} d^{\prime}$ implies $d^{\prime}<^{\prime} d$, and again $\left(x, y^{\prime}, c, d^{\prime}\right) \prec(x, y, c, d)$. In either case, the minimal choice of $(x, y, c, d)$ implies that $f_{c}(x) \cdot \omega_{x}\left(g_{d^{\prime}}\left(y^{\prime}\right)\right)=0$. Since $x \leq s$ and $x^{\prime} \leq s$, (i) implies that $\omega_{x}$ and $\omega_{x^{\prime}}$ are rigid, and $\omega_{x^{\prime}}\left(g_{d^{\prime}}\left(y^{\prime}\right)\right) \cdot f_{c}(x)=0$ follows. This proves (5.2).

Since $F G=0$, for some $n \in \mathbb{N} \cup\{0\}$ and $\left(c_{1}, d_{1}\right), \ldots,\left(c_{n}, d_{n}\right) \in T \times T \backslash\{(c, d)\}$ such that $c_{i} d_{i}=c d$,

$$
0=(F G)(c d)=F(c) G(d)+\sum_{i=1}^{n} F\left(c_{i}\right) G\left(d_{i}\right) .
$$

Since $x y \leq s$,

$$
f_{c} g_{d}+\sum_{i=1}^{n} f_{c_{i}} g_{d_{i}} \in I_{s} \Rightarrow\left(f_{c} g_{d}\right)(x y)+\sum_{i=1}^{n}\left(f_{c_{i}} g_{d_{i}}\right)(x y)=0 .
$$

By (5.2), $\left(f_{c_{i}} g_{d_{i}}\right)(x y) \cdot f_{c}(x)=0$ for every $i$; therefore,

$$
0=\left(f_{c} g_{d}\right)(x y) \cdot f_{c}(x)+\sum_{i=1}^{n}\left(f_{c_{i}} g_{d_{i}}\right)(x y) \cdot f_{c}(x)=\left(f_{c} g_{d}\right)(x y) \cdot f_{c}(x) .
$$

There exist $m \in \mathbb{N} \cup\{0\}$ and $\left(x_{1}, y_{1}\right), \ldots,\left(x_{m}, y_{m}\right) \in S \times S \backslash\{(x, y)\}$ with $x_{j} y_{j}=x y$ such that

$$
\left(f_{c} g_{d}\right)(x y)=f_{c}(x) \cdot \omega_{x}\left(g_{d}(y)\right)+\sum_{j=1}^{m} f_{c}\left(x_{j}\right) \cdot \omega_{x_{j}}\left(g_{d}\left(y_{j}\right)\right) .
$$

By (5.2), $f_{c}\left(x_{j}\right) \cdot \omega_{x_{j}}\left(g_{d}\left(y_{j}\right)\right) \cdot f_{c}(x)=0$ for every $j$; therefore, $f_{c}(x) \cdot \omega_{x}\left(g_{d}(y)\right) \cdot$ $f_{c}(x)=0$. Since (i) implies that $R$ is a reduced ring, we obtain $f_{c}(x) \cdot \omega_{x}\left(g_{d}(y)\right)=0$, a contradiction.

(ii) $\Rightarrow$ (iii) $\Rightarrow$ (iv). These are obvious.

Finally, assume that $s \neq 1$ and condition (a) or (b) holds. To prove that (iv) implies (i), assume condition (a), and let $x \in S$ satisfy $x \leq s$. If $x=1$ then $\omega_{x}$ being rigid amounts to $R$ being reduced, which is the case because $\omega_{s}$ is rigid as we will show 
in a moment. Now suppose that $x \neq 1$. Condition (a) implies that for some $n \in \mathbb{N}$, $x^{n} \leq s<x^{n+1}$. Assume that $r \in R$ satisfies $\omega_{x}(r) \cdot r=0$. Fix $t \in T \backslash\{1\}$, and put

$$
F=\overline{\mathrm{e}_{x}} 1+\overline{\mathrm{C}_{\omega_{x}}(r)} t, \quad G=\overline{\mathrm{e}_{x^{n}}} 1-\overline{\mathrm{c}_{r} \mathrm{e}_{x^{n-1}}} t \in\left(R[[S, \omega]] / I_{S}\right)[T] .
$$

Then $F G=0$. By (iv), $\mathrm{c}_{\omega_{x}(r)} \mathrm{e}_{x^{n}} \in I_{s}$, which implies that $\omega_{x}(r)=0$; by condition (a), $r=0$. From Lemma 2.5(iii) we conclude that $\omega_{x}$ is rigid.

Assume condition (b). A similar argument with $F=\overline{\mathrm{e}_{s}} 1+\overline{\mathrm{C}_{r}} t$ and $G=\overline{\mathrm{e}_{s}} 1-\overline{\mathrm{C}_{r}} t$ shows that $r^{2}=0$ implies $r=0$.

REMARK 5.5. Example 21 in [23] shows that the injectivity hypothesis in (a) of the second part of Theorem 5.4 is essential.

As a consequence of Theorem 5.4 we obtain the following generalization of [22, Theorem 3.1] and [23, Theorem 20].

COROLLARY 5.6. If $\sigma$ is an injective endomorphism of a ring $R$, and $n \geq 2$ is an integer, then the following conditions are equivalent:

(i) $R[x, \sigma] /\left(x^{n}\right)$ is Armendariz;

(ii) $R[[x, \sigma]] /\left(x^{n}\right)$ is Armendariz;

(iii) $R[x, \sigma] /\left(x^{n}\right)$ is linearly Armendariz;

(iv) $R[[x, \sigma]] /\left(x^{n}\right)$ is linearly Armendariz;

(v) $R[x, \sigma] /\left(x^{n}\right)$ is power-serieswise Armendariz;

(vi) $R[[x, \sigma]] /\left(x^{n}\right)$ is power-serieswise Armendariz;

(vii) $\sigma$ is rigid.

Proof. The equivalences (ii) $\Leftrightarrow$ (iv) $\Leftrightarrow$ (vi) $\Leftrightarrow$ (vii) follow from Theorem 5.4. The rest follows from the isomorphism $R[x, \sigma] /\left(x^{n}\right) \cong R[[x, \sigma]] /\left(x^{n}\right)$.

Note that [1, Theorem 5] is the $\sigma=1$ case of (i) $\Leftrightarrow$ (vii) in Corollary 5.6.

Let $R$ be a ring, $\left(S, \leq_{S}\right)$ and $\left(T, \leq_{T}\right)$ strictly ordered monoids, and $\omega: S \rightarrow$ $\operatorname{End}(R)$ and $v: T \rightarrow \operatorname{End}(R)$ monoid homomorphisms such that

$$
\omega_{s} \circ v_{t}=v_{t} \circ \omega_{s} \quad \forall s \in S \text { and } t \in T .
$$

It is easy to verify that the following maps are monoid homomorphisms:

- $\bar{\omega}: S \rightarrow \operatorname{End}(R[[T, v]])$, where $\bar{\omega}_{s}(g)=\omega_{S} \circ g$ for all $s \in S$ and $g \in R[[T, v]]$,

- $\bar{v}: T \rightarrow \operatorname{End}(R[[S, \omega]])$, where $\bar{v}_{t}(f)=v_{t} \circ f$ for all $t \in T$ and $f \in R[[S, \omega]]$,

- $\omega \times v: S \times T \rightarrow \operatorname{End}(R)$, where $(\omega \times v)_{(s, t)}=\omega_{s} \circ v_{t}$ for every $(s, t) \in S \times T$.

Proposition 5.7. Let $R, S, T, \omega$ and $v$ be as above. Assume that the ring $R$ is reduced, the monoids $\left(S, \leq_{S}\right)$ and $\left(T, \leq_{T}\right)$ are a.n.u.p., and for all $s \in S$ and $t \in T$ the endomorphisms $\omega_{s}$ and $v_{t}$ are injective. Then the monoid $S \times T$ is quasitotally ordered by the induced lexicographic order and the following conditions are equivalent:

(i) $\quad R$ is $(S, \omega)$-Armendariz and $(T, v)$-Armendariz;

(ii) $\quad R[[S, \omega]]$ is reduced and $(T, \bar{v})$-Armendariz; 
(iii) $R[[T, v]]$ is reduced and $(S, \bar{\omega})$-Armendariz;

(iv) $R[[S, \omega]]$ is $(T, \bar{v})$-Armendariz and $R[[T, v]]$ is $(S, \bar{\omega})$-Armendariz;

(v) $\quad R$ is $(S \times T, \omega \times v)$-Armendariz.

Proof. (i) $\Leftrightarrow$ (ii). Assume (i). Then by Theorem 4.12, for any $s \in S$ and $t \in T$ the endomorphisms $\omega_{s}$ and $v_{t}$ are rigid, and to get (ii) it suffices to show that $\bar{v}_{t}$ is a rigid endomorphism of $R[[S, \omega]]$ for every $t \in T$. To prove that, consider any $f \in R[[S, \omega]]$ with $f \bar{v}_{t}(f)=0$. Since $R$ is $(S, \omega)$-Armendariz, for any $s \in S$ we have $f(s) \cdot \omega_{s}\left(\bar{v}_{t}(f)(s)\right)=0$, that is, $f(s) \cdot \omega_{s}\left(v_{t}(f(s))\right)=0$. Since $\omega_{s}$ and $v_{t}$ are rigid, it follows that $f(s)^{2}=0$, and since $R$ is reduced, we obtain $f(s)=0$. Thus $f=0$, which completes the proof that (i) implies (ii). The converse follows directly from Theorem 4.12 and Lemma 2.7.

(i) $\Leftrightarrow$ (iii). This follows by an analogous argument.

(i) $\Leftrightarrow$ (iv). As noted above, (i) implies (ii) and (iii), so it implies (iv) as well. To prove the converse, assume (iv). Since $R$ is a subring of the $(S, \bar{\omega})$-Armendariz ring $R[[T, v]]$, Lemma 2.7 implies that $R$ is $(S, \omega)$-Armendariz. By a similar argument, $R$ is $(T, v)$-Armendariz.

(i) $\Leftrightarrow$ (v). If (i) holds, then by Theorem 4.12 the endomorphisms $\omega_{s}$ and $v_{t}$ are rigid for all $s \in S$ and $t \in T$. Hence for any $(s, t) \in S \times T$ the endomorphism $(\omega \times v)_{(s, t)} \in \operatorname{End}(R)$ is rigid, and applying Theorem 4.12, we obtain (v). That (v) implies (i) is an immediate consequence of Lemma 2.7.

COROLlary 5.8. Let $R$ be a reduced ring, $\left(S, \leq_{S}\right)$ and $\left(T, \leq_{T}\right)$ strictly ordered a.n.u.p. monoids, and $\omega: S \rightarrow \operatorname{End}(R)$ a monoid homomorphism such that $\omega_{s}$ is injective for every $s \in S$. Then $R$ is $(S, \omega)$-Armendariz if and only if $R[[T, 1]]$ is $(S, \bar{\omega})$-Armendariz.

PROOF. Applying the implications (vi) $\Rightarrow$ (ii) and (vi) $\Rightarrow$ (iv) of Theorem 4.12, we deduce that $R$ is $(T, 1)$-Armendariz and $R[[T, 1]]$ is reduced. Now the corollary follows from the equivalence (i) $\Leftrightarrow$ (iii) of Proposition 5.7.

Applying Corollary 5.8 when $T$ is the additive monoid of nonnegative integers with the trivial order, the additive monoid of nonnegative integers with the usual order, the additive group of integers with the trivial order and the additive group of integers with the usual order, respectively, we obtain the following corollary.

COROLlaRY 5.9. Let $R$ be a reduced ring, $(S, \leq)$ a strictly ordered a.n.u.p. monoid, and $\omega: S \rightarrow \operatorname{End}(R)$ a monoid homomorphism such that $\omega_{s}$ is injective for every $s \in S$. Then the following are equivalent:

(i) the ring $R$ is $(S, \omega)$-Armendariz;

(ii) the polynomial ring $R[x]$ is $(S, \bar{\omega})$-Armendariz;

(iii) the power series ring $R[[x]]$ is $(S, \bar{\omega})$-Armendariz;

(iv) the Laurent polynomial ring $R\left[x, x^{-1}\right]$ is $(S, \bar{\omega})$-Armendariz;

(v) the Laurent series ring $R\left[\left[x, x^{-1}\right]\right]$ is $(S, \bar{\omega})$-Armendariz.

A special case of Corollary 5.9 is the following result of Chen and Tong (with a slight change of notation). 
Corollary 5.10 [8, Proposition 6]. Let $R$ be a reduced ring and $\sigma$ a monomorphism of $R$. Then $R$ is $\sigma$-skew Armendariz if and only if $R[x]$ is $\bar{\sigma}$-skew Armendariz.

\section{Uniserial rings are Armendariz}

Recall that a module $M$ is said to be uniserial if the submodule lattice of $M$ is totally ordered; a ring $R$ is said to be right (left) uniserial if the module $R_{R}\left({ }_{R} R\right)$ is uniserial. A ring $R$ is said to be $d u o$ if every one-sided ideal of $R$ is an ideal.

PROPOSITION 6.1. Let $R$ be a right uniserial ring, $(S, \leq)$ a strictly ordered a.n.u.p. monoid and $\omega: S \rightarrow \operatorname{End}(R)$ a monoid homomorphism. Assume the Jacobson radical $\operatorname{rad}(R)$ is $S$-invariant. Suppose that $f, g \in R[[S, \omega]]$ satisfy $f g=0$ and that there exist $s_{0}, t_{0} \in S$ such that $f(s) \cdot \omega_{s}(g(t)) \in f\left(s_{0}\right) \cdot \omega_{s_{0}}\left(g\left(t_{0}\right)\right) R$ for all $s, t \in S$. Then $f(s) \cdot \omega_{s}(g(t))=0$ for all $s, t \in S$.

PROOF. Let

$$
\begin{aligned}
& X=\left\{x \in S:(\exists u \in S)(\forall s, t \in S) f(s) \cdot \omega_{s}(g(t)) \in f(x) \cdot \omega_{x}(g(u)) R\right\}, \\
& Y=\left\{y \in S:(\exists v \in S)(\forall s, t \in S) f(s) \cdot \omega_{s}(g(t)) \in f(v) \cdot \omega_{v}(g(y)) R\right\} .
\end{aligned}
$$

If $X \nsubseteq \operatorname{supp}(f)$ or $Y \nsubseteq \operatorname{supp}(g)$, then clearly $f(s) \cdot \omega_{s}(g(t))=0$ for all $s, t \in S$. Thus, we will assume that $X \subseteq \operatorname{supp}(f)$ and $Y \subseteq \operatorname{supp}(g)$. The sets $X$ and $Y$ are artinian, narrow, and nonempty (because $s_{0} \in X$ and $t_{0} \in Y$ ). Since $(S, \leq)$ is a.n.u.p., there exist $x_{0} \in X$ and $y_{0} \in Y$ such that $x_{0} y_{0}$ is a u.p. element of $X Y$. Since $x_{0} \in X$ and $y_{0} \in Y$, for some $u_{0}, v_{0} \in S$ and all $s, t \in S$,

$$
f(s) \cdot \omega_{s}(g(t)) \in f\left(x_{0}\right) \cdot \omega_{x_{0}}\left(g\left(u_{0}\right)\right) R
$$

and

$$
f(s) \cdot \omega_{s}(g(t)) \in f\left(v_{0}\right) \cdot \omega_{v_{0}}\left(g\left(y_{0}\right)\right) R .
$$

Assume that $g\left(u_{0}\right) R \subseteq g\left(y_{0}\right) R$. Then $\omega_{x_{0}}\left(g\left(u_{0}\right)\right) R \subseteq \omega_{x_{0}}\left(g\left(y_{0}\right)\right) R$, and (6.1) implies that

$$
f(s) \cdot \omega_{s}(g(t)) \in f\left(x_{0}\right) \cdot \omega_{x_{0}}\left(g\left(y_{0}\right)\right) R \quad \forall s, t \in S .
$$

Since $f g=0$,

$$
0=(f g)\left(x_{0} y_{0}\right)=f\left(x_{0}\right) \cdot \omega_{x_{0}}\left(g\left(y_{0}\right)\right)+\sum_{(p, q) \in \mathrm{X}_{x_{0} y_{0}}(f, g) \backslash\left\{\left(x_{0}, y_{0}\right)\right\}} f(p) \cdot \omega_{p}(g(q)) .
$$

Note that if $(p, q) \in S \times S \backslash\left\{\left(x_{0}, y_{0}\right)\right\}$ satisfies $p q=x_{0} y_{0}$, then since $x_{0} y_{0}$ is a u.p. element of $X Y$, we have $p \notin X$ or $q \notin Y$. In either case, (6.3) implies that $f\left(x_{0}\right) \cdot \omega_{x_{0}}\left(g\left(y_{0}\right)\right) R \nsubseteq f(p) \cdot \omega_{p}(g(q)) R$; therefore, since $R$ is right uniserial,

$$
f(p) \cdot \omega_{p}(g(q)) \in f\left(x_{0}\right) \cdot \omega_{x_{0}}\left(g\left(y_{0}\right)\right) \cdot \operatorname{rad}(R) .
$$


Thus, (6.4) and (6.5) imply that for some $r \in \operatorname{rad}(R)$,

$$
0=f\left(x_{0}\right) \cdot \omega_{x_{0}}\left(g\left(y_{0}\right)\right)+f\left(x_{0}\right) \cdot \omega_{x_{0}}\left(g\left(y_{0}\right)\right) r=f\left(x_{0}\right) \cdot \omega_{x_{0}}\left(g\left(y_{0}\right)\right)(1+r),
$$

which implies that $f\left(x_{0}\right) \cdot \omega_{x_{0}}\left(g\left(y_{0}\right)\right)=0$. By (6.3), $f(s) \cdot \omega_{s}(g(t))=0$ for all $s, t \in S$ in this case.

We are left with the case where $g\left(u_{0}\right) R \nsubseteq g\left(y_{0}\right) R$. Since $R$ is right uniserial, $g\left(y_{0}\right) R \subseteq g\left(u_{0}\right) \cdot \operatorname{rad}(R)$. Choose $r \in \operatorname{rad}(R)$ such that $g\left(y_{0}\right)=g\left(u_{0}\right) r$. Then

$$
\omega_{v_{0}}\left(g\left(y_{0}\right)\right)=\omega_{v_{0}}\left(g\left(u_{0}\right)\right) \cdot \omega_{v_{0}}(r) \in \omega_{v_{0}}\left(g\left(u_{0}\right)\right) \cdot \operatorname{rad}(R),
$$

and from (6.2) we obtain

$$
f(s) \cdot \omega_{s}(g(t)) \in f\left(v_{0}\right) \cdot \omega_{v_{0}}\left(g\left(u_{0}\right)\right) \cdot \operatorname{rad}(R) \quad \forall s, t \in S .
$$

Applying (6.6) with $s=v_{0}$ and $t=u_{0}$, we obtain $f\left(v_{0}\right) \cdot \omega_{v_{0}}\left(g\left(u_{0}\right)\right)=0$, and another application of (6.6) yields $f(s) \cdot \omega_{s}(g(t))=0$ for all $s, t \in S$, which completes the proof.

COROLlary 6.2. Let $R$ be a right or left uniserial ring and $S$ a u.p. monoid. Then $R$ is Armendariz relative to $S$.

COROLlary 6.3. Every right or left uniserial ring is Armendariz.

COROLLARY 6.4. Let $R$ be a right noetherian, right uniserial ring, $(S, \leq)$ a strictly ordered a.n.u.p. monoid, and $\omega: S \rightarrow \operatorname{End}(R)$ a monoid homomorphism. Assume that the Jacobson radical $\operatorname{rad}(R)$ is $S$-invariant. Then $R$ is $(S, \omega)$-Armendariz.

The following example shows that in Corollary 6.4 the noetherian hypothesis is essential.

EXAMPLE 6.5. Let $U$ be a commutative uniserial domain and $M$ a divisible, uniserial $U$-module that is not torsion-free. Such a pair $U$ and $M$ exist under ZFC by [10, Lemma 7]. Choose $u \in U \backslash\{0\}$ and $m \in M \backslash\{0\}$ such that $u m=0$. Put $m_{0}=m$; then by divisibility of $M$ we can define a sequence $\left\{m_{0}, m_{1}, m_{2}, \ldots\right\}$ of elements of $M$ such that $m_{n-1}=u m_{n}$ for all $n \in \mathbb{N}$. Define $R=U \oplus M$ as an additive group, with multiplication given by

$$
\left(v_{1}, n_{1}\right)\left(v_{2}, n_{2}\right)=\left(v_{1} v_{2}, v_{1} n_{2}+v_{2} n_{1}\right) .
$$

Since $M$ is divisible and $U$ and $M$ are uniserial, it follows that $R$ is a commutative uniserial ring. Nevertheless, $R$ is not $(S, 1)$-Armendariz for $S=\mathbb{N} \cup\{0\}$ with the standard order $\leq$. Indeed, this ring $R[[S, 1]]$ is isomorphic to the power series ring $R[[x]]$, and for the power series $f, g \in R[[x]]$ defined by

$$
f=(u, 0)-(1,0) x, \quad g=\left(0, m_{0}\right)+\left(0, m_{1}\right) x+\left(0, m_{2}\right) x^{2}+\cdots,
$$

we have $f g=0$, although $(1,0)\left(0, m_{0}\right) \neq 0$ in $R$. 


\section{Triangular matrix rings}

In [13], Hong et al. obtained a wide range of detailed results on the skew Armendariz condition in triangular matrix rings. We will now prove a proposition that unifies two of the results in [13] within the context of skew generalized power series rings.

Let $R$ be a ring, $S$ a monoid, $\omega: S \rightarrow \operatorname{End}(R)$ a monoid homomorphism, $n$ a positive integer, and $\mathbb{M}_{n}(R)$ the ring of $n \times n$ matrices over $R$. For $s \in S$, let $\bar{\omega}_{s}: \mathbb{M}_{n}(R) \rightarrow \mathbb{M}_{n}(R)$ be the map obtained by applying $\omega_{s}$ to every entry of a given matrix in $\mathbb{M}_{n}(R)$. We thereby obtain a monoid homomorphism $\bar{\omega}: S \rightarrow \operatorname{End}\left(\mathbb{M}_{n}(R)\right)$. Given any subring $T \subseteq \mathbb{M}_{n}(R)$ that is invariant under the action of $S$, we have a monoid homomorphism, which (in a slight abuse of notation) we will also denote by $\bar{\omega}: S \rightarrow \operatorname{End}(T)$, obtained by restricting the homomorphisms $\bar{\omega}_{s}$ to $T$.

PROposition 7.1. Let $R$ be a ring, $(S, \leq)$ a strictly ordered monoid, $\omega: S \rightarrow$ $\operatorname{End}(R)$ a monoid homomorphism, and $n$ any positive integer. Define a subring $T$ of $\mathbb{M}_{n}(R)$ as follows:

$$
T=\left\{\left(\begin{array}{cccccc}
a & b_{1} & b_{2} & b_{3} & \cdots & b_{n-1} \\
0 & a & c_{1} & c_{2} & \cdots & c_{n-2} \\
0 & 0 & a & 0 & \cdots & 0 \\
\vdots & \vdots & \ddots & \ddots & \ddots & \vdots \\
0 & 0 & \cdots & 0 & a & 0 \\
0 & 0 & \cdots & 0 & 0 & a
\end{array}\right): a, b_{1}, \ldots, b_{n-1}, c_{1}, \ldots, c_{n-2} \in R\right\}
$$

Suppose $R$ is reduced, and $\omega_{s}$ is injective for every $s \in S$. Then $R$ is $(S, \omega)$-Armendariz if and only if $T$ is $(S, \bar{\omega})$-Armendariz.

Proof. If $T$ is $(S, \bar{\omega})$-Armendariz then $R$ is $(S, \omega)$-Armendariz by Lemma 2.7.

Conversely, assume that $R$ is $(S, \omega)$-Armendariz. Suppose that $f, g \in T[[S, \bar{\omega}]]$ satisfy $f g=0$. Now, $f$ and $g$ are functions from $S$ to $T$ with artinian, narrow support. Given any $s \in S$,

$$
f(s)=\left(\begin{array}{cccccc}
f_{1,1}(s) & f_{1,2}(s) & f_{1,3}(s) & f_{1,4}(s) & \cdots & f_{1, n}(s) \\
0 & f_{2,2}(s) & f_{2,3}(s) & f_{2,4}(s) & \cdots & f_{2, n}(s) \\
0 & 0 & f_{3,3}(s) & 0 & \cdots & 0 \\
\vdots & \vdots & \ddots & \ddots & \ddots & \vdots \\
0 & 0 & \ldots & 0 & f_{n-1, n-1}(s) & 0 \\
0 & 0 & \cdots & 0 & 0 & f_{n, n}(s)
\end{array}\right)
$$


and

$$
g(s)=\left(\begin{array}{cccccc}
g_{1,1}(s) & g_{1,2}(s) & g_{1,3}(s) & g_{1,4}(s) & \ldots & g_{1, n}(s) \\
0 & g_{2,2}(s) & g_{2,3}(s) & g_{2,4}(s) & \ldots & g_{2, n}(s) \\
0 & 0 & g_{3,3}(s) & 0 & \ldots & 0 \\
\vdots & \vdots & \ddots & \ddots & \ddots & \vdots \\
0 & 0 & \ldots & 0 & g_{n-1, n-1}(s) & 0 \\
0 & 0 & \ldots & 0 & 0 & g_{n, n}(s)
\end{array}\right)
$$

where each $f_{i, j}$ and each $g_{i, j}$ is a function from $S$ to $R$, and $f_{1,1}=f_{2,2}=\cdots=f_{n, n}$ and $g_{1,1}=g_{2,2}=\cdots=g_{n, n}$. Since $\operatorname{supp}\left(f_{i, j}\right) \subseteq \operatorname{supp}(f)$ and $\operatorname{supp}\left(g_{i, j}\right) \subseteq \operatorname{supp}(g)$, each $f_{i, j}$ and each $g_{i, j}$ has artinian, narrow support. Hence $f_{i, j}, g_{i, j} \in R[[S, \omega]]$.

For every $s \in S$,

$$
0=(f g)(s)=\sum_{(x, y) \in \mathrm{X}_{s}(f, g)} f(x) \cdot \bar{\omega}_{x}(g(y)),
$$

and therefore, for all $i, j$,

$$
\begin{aligned}
0 & =\sum_{k} \sum_{(x, y) \in \mathrm{X}_{s}(f, g)} f_{i, k}(x) \cdot \omega_{x}\left(g_{k, j}(y)\right) \\
& =\sum_{k} \sum_{(x, y) \in \mathrm{X}_{s}\left(f_{i, k}, g_{k, j}\right)} f_{i, k}(x) \cdot \omega_{x}\left(g_{k, j}(y)\right) \\
& =\sum_{k}\left(f_{i, k} g_{k, j}\right)(s) .
\end{aligned}
$$

In the ring $R[[S, \omega]]$, which by Theorem 4.12 is reduced, the following equations hold:

$$
\begin{gathered}
f_{1,1} g_{1,1}=0 \\
f_{1,1} g_{1,2}+f_{1,2} g_{1,1}=0 \\
f_{1,1} g_{2, i}+f_{2, i} g_{1,1}=0 \text { for } i=3,4, \ldots, n, \\
f_{1,1} g_{1, i}+f_{1,2} g_{2, i}+f_{1, i} g_{1,1}=0 \text { for } i=3,4, \ldots, n .
\end{gathered}
$$

Reduced rings are symmetric in the terminology of [28]: whenever a product of elements equals 0, any permutation of the factors also has product 0. Equations (7.1) and (7.2) therefore yield

$$
0=f_{1,1} g_{1,2} f_{1,1}+f_{1,2} g_{1,1} f_{1,1}=f_{1,1} g_{1,2} f_{1,1},
$$

and $\left(f_{1,1} g_{1,2}\right)^{2}=0$ implies that $f_{1,1} g_{1,2}=f_{1,2} g_{1,1}=0$. Applying the same argument to Equation (7.3) yields $f_{1,1} g_{2, i}=f_{2, i} g_{1,1}=0$, and then applying it to Equation (7.4) yields $f_{1,1} g_{1, i}=f_{1,2} g_{2, i}+f_{1, i} g_{1,1}=0$. Since $f_{1,2} g_{1,1}=0$, from $g_{1,1} f_{1,2} g_{2, i}+$ $g_{1,1} f_{1, i} g_{1,1}=0$ we likewise obtain $f_{1, i} g_{1,1}=f_{1,2} g_{2, i}=0$. Thus, every summand in Equations (7.1)-(7.4) equals 0 in $R[[S, \omega]]$. 
By hypothesis, $R$ is $(S, \omega)$-Armendariz. Therefore, for all $s, t \in S$,

$$
\begin{gathered}
f_{1,1}(s) \cdot \omega_{s}\left(g_{1,1}(t)\right)=0, \\
f_{1,1}(s) \cdot \omega_{s}\left(g_{1,2}(t)\right)=f_{1,2}(s) \cdot \omega_{s}\left(g_{1,1}(t)\right)=0, \\
f_{1,1}(s) \cdot \omega_{s}\left(g_{2, i}(t)\right)=f_{2, i}(s) \cdot \omega_{s}\left(g_{1,1}(t)\right)=0 \quad \text { for } i=3,4, \ldots, n, \\
f_{1,1}(s) \cdot \omega_{s}\left(g_{1, i}(t)\right)=f_{1,2}(s) \cdot \omega_{s}\left(g_{2, i}(t)\right) \\
=f_{1, i}(s) \cdot \omega_{s}\left(g_{1,1}(t)\right)=0 \quad \text { for } i=3,4, \ldots, n .
\end{gathered}
$$

In particular, for all $i, j$,

$$
\sum_{k} f_{i, k}(s) \cdot \omega_{s}\left(g_{k, j}(t)\right)=0
$$

and therefore $f(s) \cdot \bar{\omega}_{s}(g(t))=0$ for all $s, t \in S$. This proves that $T$ is $(S, \bar{\omega})$ Armendariz.

The $n=2$ case of the 'only if' part of Proposition 7.1, in conjunction with Example 2.2(ii), recovers [13, Proposition 15]. Analogously, the $n=3$ case recovers [13, Proposition 17]. The fact that when $n \leq 3$ the ring of upper triangular $n \times n$ matrices with constant diagonal over a $\sigma$-rigid ring is $\bar{\sigma}$-skew Armendariz, as pointed out by Hong et al. in [13, Example 18], does not generalize to $n=4$. The fatal flaw can be traced to the nonzero $(3,4)$-entry of the matrix! Proposition 7.1 demonstrates a different direction in which a viable generalization is possible.

In the proof of the next result we will need the following criterion for $S$-rigidity of subrings of an $(S, \omega)$-Armendariz ring.

LEMMA 7.2. Let $T$ be a ring, $(S, \leq)$ an ordered monoid, and $\omega: S \rightarrow \operatorname{End}(T)$ a monoid homomorphism. Suppose that $T$ is linearly $(S, \omega)$-Armendariz, suppose that $R$ is an $S$-invariant, $S$-compatible subring of $T$, and suppose that there exists $b \in T$ with the property that $b \neq b^{2}=0, R \cap \operatorname{ann}_{r}^{T}(b)=\{0\}$, and that there exists $s \in S \backslash\{1\}$ such that for every $r \in R, b r=r \omega_{s}(b)$. Then $R$ is $S$-rigid.

Proof. By Lemma 2.5(iii), it suffices to show that $R$ is reduced. Suppose $a \in R$ satisfies $a^{2}=0$. Put

$$
f=\mathrm{c}_{b}+\mathrm{c}_{a} \mathrm{e}_{s}, \quad g=\mathrm{c}_{b}+\mathrm{c}_{-a} \mathrm{e}_{s} \text { in } T[[S, \omega]] .
$$

Using the $S$-compatibility of $R$ and the hypotheses on $b$, we find that $f g=0$. Since $T$ is linearly $(S, \omega)$-Armendariz, $-b a=0$, hence $a \in R \cap \operatorname{ann}_{r}^{T}(b)=\{0\}$.

COROLlary 7.3. Let $R$ be a ring, $(S, \leq)$ a nontrivial strictly ordered monoid, $\omega: S \rightarrow \operatorname{End}(R)$ a monoid homomorphism, and $n \geq 2$ an integer. Let $T \subset \mathbb{M}_{n}(R)$ be the subring defined in Proposition 7.1. Suppose $R$ is $S$-compatible. Then $R$ is reduced and $(S, \omega)$-Armendariz if and only if $T$ is $(S, \bar{\omega})$-Armendariz.

PROOF. For the 'only if' part, apply Lemma 2.5(i) and Proposition 7.1.

For the 'if' part, apply Lemma 7.2 ( $b$ can be taken to be the matrix with a 1 in the $(1,2)$-position and 0s elsewhere) and Lemma 2.7. 
Corollary 7.3 shows that the hypothesis in Proposition 7.1 that $R$ be reduced is indispensable. Clearly, the conclusion of Proposition 7.1 fails without the hypothesis that every $\omega_{s}$ be injective. So Proposition 7.1 is 'sharp' in some sense.

We note that a more general class of upper triangular matrix rings, of the sort analysed by Birkenmeier and Park in [5], affords a skew generalized power series construction. Let $A$ and $B$ be rings, let $M$ be an $(A, B)$-bimodule, and let

$$
T=\left(\begin{array}{cc}
A & M \\
0 & B
\end{array}\right)
$$

Suppose that $(S, \leq)$ is an ordered monoid, and let $\omega: S \rightarrow \operatorname{End}(A)$ and $v: S \rightarrow$ $\operatorname{End}(B)$ be monoid homomorphisms. Assume that $\mu: S \rightarrow \operatorname{End}_{\mathbb{Z}}(M)$ is a monoid homomorphism with the property that for every $s \in S$,

$$
\mu_{s}(a m)=\omega_{s}(a) \mu_{s}(m) \quad \text { and } \quad \mu_{s}(m b)=\mu_{s}(b) v_{s}(b)
$$

for all $a \in A, b \in B$, and $m \in M$. (In the case where $\omega(S) \subseteq \operatorname{Aut}(A)$ and $v(S) \subseteq$ $\operatorname{Aut}(B)$, in particular when $S$ is a group, this map $\mu_{s}: M \rightarrow M$ is a morphism in the category of $(A, B)$-bimodules where the left action of $A$ is twisted by $\omega_{s}^{-1}$ and the right action of $B$ by $v_{s}^{-1}$.) Now if we define

$$
\eta_{s}\left(\left(\begin{array}{cc}
a & m \\
0 & b
\end{array}\right)\right)=\left(\begin{array}{cc}
\omega_{s}(a) & \mu_{s}(m) \\
0 & v_{s}(b)
\end{array}\right)
$$

we obtain a monoid homomorphism $\eta: S \rightarrow \operatorname{End}(T)$.

For example, given a ring $R$, a strictly ordered monoid $(S, \leq)$, and a monoid homomorphism $\omega: S \rightarrow \operatorname{End}(R)$, we can construct a skew generalized power series ring over the ring of upper triangular $n \times n$ matrices with entries in $R$ by composing $\omega: S \rightarrow \operatorname{End}(R)$ with the diagonal embedding $\operatorname{End}(R) \rightarrow \operatorname{End}\left(\prod_{i=1}^{n-1} R\right)$, letting $A=$ $\prod_{i=1}^{n-1} R, B=R$, and $v=\omega$, taking $M=\bigoplus_{i=1}^{(n-1) n / 2} R$ to correspond to the strictly upper triangular matrix positions, and defining $\mu_{s}: M \rightarrow M$ by applying $\omega_{s}$ to every coordinate. In this case $\eta=\bar{\omega}$, the homomorphism defined prior to Proposition 7.1.

Proposition 7.1 certainly cannot be extended to the ring $T$. For example, if $T$ is the ring of upper triangular $n \times n$ matrices over any ring $R$, where $n \geq 2$, then $T$ is not Armendariz. This observation serves to complement [13, Example 18], circumscribing the possible generalizations of [13, Propositions 15 and 17].

The unified approach to Armendariz-like rings developed in this paper can also be applied to some other generalizations of Armendariz rings, for example, to quasiArmendariz rings and their various modifications. Furthermore, it can be used to unify numerous results on Baer rings, quasi-Baer rings, right Rickart rings (also known as right p.p.-rings), and so on. We will present results in these directions in forthcoming work. 


\section{Acknowledgement}

Parts of this work were completed during a visit by the first author to the Bialystok University of Technology; he is grateful for the generous hospitality and support he received.

\section{References}

[1] D. D. Anderson and V. Camillo, 'Armendariz rings and Gaussian rings', Comm. Algebra 26(7) (1998), 2265-2272.

[2] S. Annin, 'Associated primes over skew polynomial rings', Comm. Algebra 30(5) (2002), $2511-2528$.

[3] E. P. Armendariz, 'A note on extensions of Baer and P.P.-rings', J. Aust. Math. Soc. 18 (1974), 470-473.

[4] G. F. Birkenmeier, H. E. Heatherly and E. K. Lee, 'Completely prime ideals and associated radicals', in: Ring Theory (Granville, $\mathrm{OH}, 1992$ ), (eds. S. K. Jain and S. T. Rizvi) (World Scientific, Singapore, 1993), pp. 102-129.

[5] G. F. Birkenmeier and J. K. Park, 'Triangular matrix representations of ring extensions', J. Algebra $\mathbf{2 6 5}(2)$ (2003), 457-477.

[6] V. Camillo, W. K. Nicholson and M. F. Yousif, 'Ikeda-Nakayama rings', J. Algebra 226 (2000), 1001-1010.

[7] V. Camillo and P. P. Nielsen, 'McCoy rings and zero-divisors', J. Pure Appl. Algebra 212(3) (2008), 599-615.

[8] W. Chen and W. Tong, 'A note on skew Armendariz rings', Comm. Algebra 33 (2005), 1137-1140.

[9] S. P. Farbman, 'The unique product property of groups and their amalgamated free products', J. Algebra 178(3) (1995), 962-990.

[10] L. Fuchs and S. Shelah, 'Kaplansky's problem on valuation rings', Proc. Amer. Math. Soc. 105(1) (1989), 25-30.

[11] E. Hashemi and A. Moussavi, 'Polynomial extensions of quasi-Baer rings', Acta Math. Hungar. 107(3) (2005), 207-224.

[12] Y. Hirano, 'On annihilator ideals of a polynomial ring over a noncommutative ring', J. Pure Appl. Algebra 168 (2002), 45-52.

[13] C. Y. Hong, N. K. Kim and T. K. Kwak, 'On skew Armendariz rings', Comm. Algebra 31(1) (2003), 103-122.

[14] C. Huh, H. K. Kim and Y. Lee, 'Questions on 2-primal rings', Comm. Algebra 26(2) (1998), 595-600.

[15] C. Huh, Y. Kim and A. Smoktunowicz, 'Armendariz rings and semicommutative rings', Comm. Algebra 30(2) (2002), 751-761.

[16] A. Jategaonkar, 'Skew polynomial rings over orders in Artinian rings', J. Algebra 21 (1972), $51-59$.

[17] N. K. Kim and Y. Lee, 'Armendariz rings and reduced rings', J. Algebra 223(2) (2000), 477-488.

[18] N. K. Kim, K. H. Lee and Y. Lee, 'Power series rings satisfying a zero divisor property', Comm. Algebra 34(6) (2006), 2205-2218.

[19] M. T. Kosan, 'The Armendariz module and its application to the Ikeda-Nakayama module', Int. J. Math. Math. Sci. 2006 (2006), 7. Article ID 35238.

[20] J. Krempa, 'Some examples of reduced rings', Algebra Colloq. 3(4) (1996), 289-300.

[21] T. Y. Lam, A First Course in Noncommutative Rings, Graduate Texts in Mathematics, 131 (Springer, New York, 1991).

[22] T.-K. Lee and T.-L. Wong, 'On Armendariz rings', Houston J. Math. 29(3) (2003), 583-593.

[23] T.-K. Lee and Y. Zhou, 'A unified approach to the Armendariz property of polynomial rings and power series rings', Colloq. Math. 113(1) (2008), 151-168. 
[24] Z. Liu, 'Special properties of rings of generalized power series', Comm. Algebra 32(8) (2004), 3215-3226.

[25] Z. Liu, 'Armendariz rings relative to a monoid', Comm. Algebra 33(3) (2005), 649-661.

[26] G. Marks, 'Direct product and power series formations over 2-primal rings', in: Advances in Ring Theory, 26, (eds. S. K. Jain and S. T. Rizvi) (Birkhäuser, Boston, MA, 1997), pp. 239-245.

[27] G. Marks, 'Reversible and symmetric rings', J. Pure Appl. Algebra 174(3) (2002), 311-318.

[28] G. Marks, 'A taxonomy of 2-primal rings', J. Algebra 266(2) (2003), 494-520.

[29] G. Marks, R. Mazurek and M. Ziembowski, 'A new class of unique product monoids with applications to ring theory', Semigroup Forum 78(2) (2009), 210-225.

[30] J. Matczuk, 'A characterization of $\sigma$-rigid rings', Comm. Algebra 32(11) (2004), 4333-4336.

[31] R. Mazurek and M. Ziembowski, 'On von Neumann regular rings of skew generalized power series', Comm. Algebra 36(5) (2008), 1855-1868.

[32] A. Moussavi and E. Hashemi, 'On $(\alpha, \delta)$-skew Armendariz rings', J. Korean Math. Soc. 42(2) (2005), 353-363.

[33] C. S. Nagore and M. Satyanarayana, 'On naturally ordered semigroups', Semigroup Forum 18(2) (1979), 95-103.

[34] A. R. Nasr-Isfahani and A. Moussavi, 'On classical quotient rings of skew Armendariz rings', Int. J. Math. Math. Sci. 2007 (2007), 7. Article ID 61549.

[35] J. Okniński, Semigroup Algebras, Monographs and Textbooks in Pure and Applied Mathematics, 138 (Marcel Dekker, New York, 1991).

[36] E. R. Puczyłowski, 'Questions related to Koethe's nil ideal problem', in: Algebra and its Applications, Contemporary Mathematics, 419 (American Mathematical Society, Providence, RI, 2006), pp. 269-283.

[37] M. B. Rege and S. Chhawchharia, 'Armendariz rings', Proc. Japan Acad. Ser. A Math. Sci. 73 (1997), 14-17.

GREG MARKS, Department of Mathematics and Computer Science,

St. Louis University, St. Louis, MO 63103, USA

e-mail: marks@slu.edu

RYSZARD MAZUREK, Faculty of Computer Science,

Bialystok University of Technology, Wiejska 45A, 15-351 Bialystok, Poland

e-mail: mazurek@pb.bialystok.pl

MICHAŁ ZIEMBOWSKI, Maxwell Institute of Sciences, School of Mathematics, University of Edinburgh, James Clerk Maxwell Building, King's Buildings, Mayfield Road, Edinburgh EH9 3JZ, UK

e-mail: m.ziembowski@wp.pl 